\title{
ANALYTIC EXTENSIONS OF DIFFERENTIABLE FUNCTIONS DEFINED IN CLOSED SETS*
}

\author{
BY \\ HASSLER WHITNEY†
}

\section{Differentiable FunCtions IN CLOSED SETS}

1. Introduction. Let $A$ be a closed set, bounded or unbounded, in euclidean $n$-space $E$, and let $f(x)$ be a function defined and continuous in $A$. It is well known that this function can be extended so as to be continuous throughout $E$. $\ddagger$ If $A$ satisfies certain conditions, the solution of the Dirichlet problem is a function harmonic in $E-A$ and taking on the given boundary values in $A$. Two questions which arise are the following: Is there always a function differentiable, or perhaps analytic, in $E-A$, and taking on the given values in $A$ ? If the given function $f(x)$ is in some sense differentiable in $A$, can the extension $F(x)$ be made differentiable to the same order throughout $E$ ?

These questions are answered in the affirmative in Theorem I. We use a definition of the derivatives of a function in a general set which arises naturally from a consideration of Taylor's formula. In Part II, a differentiable extension of $f(x)$ is found, whether $f(x)$ is differentiable to finite or infinite order. Part III is devoted to some general approximation theorems. It is well known that a continuous function in a bounded closed set can be approximated uniformly (together with any finite number of derivatives) by polynomials; we show that functions defined in open sets may be approximated (together with derivatives) by analytic functions, the approximation being closer and closer as we approach the boundary of the set. This theorem, together with the results of Part II, furnish an immediate proof of Theorem I. In Part IV we give some extensions of Theorem I; in particular, we show that

\footnotetext{
* Presented to the Society, December 29, 1932; received by the editors March 29, 1933, and, after revision, May 2, 1933.

$\dagger$ National Research Fellow.

† See references in a paper by P. Urysohn, Mathematische Annalen, vol. 94 (1925), p. 293, footnote 51 .

A continuous extension the author has not seen in the literature may be given as follows; we assume for simplicity that $A$ is bounded. Let $h(r)(r \geqq 0)$ be a continuous and monotone increasing function such that $h(0)=0$, and if $x$ and $y$ are any two points of $A$ whose distance apart is $r_{x y}$, then $|f(x)-f(y)| \leqq h\left(r_{x y}\right)$. For any points $x$ of $E$ and $y$ of $A$, set $H(x, y)=f(y)-h\left(r_{x y}\right)$; then if $x$ is in $A$, $H(x, y) \leqq f(x)$. The continuous extension of $f(x)$ is $F(x)$, which at each point $x$ of $E$ equals the maximum of $H(x, y)$ as $y$ varies over $A$.
} 
the extension of $f(x)$ may be made analytic at the isolated points of $A$. Theorem III includes all preceding results but Lemma 7 .

2. Notations. We shall write all equations involving $n$ variables as if there were but a single variable present. For instance, we write

$$
\begin{gathered}
f_{0}(x) \text { for } f_{0} \ldots 0\left(x_{1}, \cdots, x_{n}\right), \\
D_{k} f\left(x^{\prime}\right) \text { for } \frac{\partial^{k_{1}+\cdots+k_{n}}}{\partial x_{1} k_{1} \cdots \partial x_{n} k_{n}} f\left(x_{1}^{\prime}, \cdots, x_{n}{ }^{\prime}\right), \\
\left(\begin{array}{l}
k \\
l
\end{array}\right) \text { for }\left(\begin{array}{c}
k_{1} \\
l_{1}
\end{array}\right) \cdots\left(\begin{array}{c}
k_{n} \\
l_{n}
\end{array}\right),
\end{gathered}
$$

etc. For any $n$-fold subscript $k$, we put

$$
\sigma_{k}=k_{1}+\cdots+k_{n} \text {. }
$$

Note that $\sigma_{k+l}=\sigma_{k}+\sigma_{l} . r_{x y}$ will always denote the distance between $x$ and $y$ (unless $x$ and $y$ are complex). As an example, (3.1) below is short for

$$
\begin{aligned}
& f_{k_{1}} \cdots k_{n}\left(x_{1}^{\prime}, \cdots, x_{n}^{\prime}\right) \\
&= \sum_{\substack{l_{1}+\cdots+l_{n} \\
\leqq m-\left(k_{1}+\cdots+k_{n}\right)}} \frac{f_{k_{1}+l_{1}, \cdots, k_{n}+l_{n}}\left(x_{1}, \cdots, x_{n}\right)}{l_{1} ! \cdots l_{n} !}\left(x_{1}^{\prime}-x_{1}\right)^{l_{1}} \cdots\left(x_{n}{ }^{\prime}-x_{n}\right)^{l_{n}} \\
& \quad+R_{k_{1}} \cdots k_{n}\left(x_{1}^{\prime}, \cdots, x_{n}{ }^{\prime} ; x_{1}, \cdots, x_{n}\right) .
\end{aligned}
$$

3. Differentiable functions in subsets of $E$. Let $f(x)$ be defined in the set $A$, and let $m$ be an integer $\geqq 0$. We say $f(x)=f_{0}(x)$ is of class $C^{m}$ in $A$ in terms of the functions $f_{k}(x)\left(\sigma_{k} \leqq m\right)$ if the functions $f_{k}(x)$ are defined in $A$ for all $n$-fold subscripts $k$ with $\sigma_{k} \leqq m$, and

$$
f_{k}\left(x^{\prime}\right)=\sum_{\sigma_{l} \leqq m-\sigma_{k}} \frac{f_{k+l}(x)}{l !}\left(x^{\prime}-x\right)^{l}+R_{k}\left(x^{\prime} ; x\right)
$$

for each $f_{k}(x)\left(\sigma_{k} \leqq m\right)$, where $R_{k}\left(x^{\prime} ; x\right)$ has the following property. Given any point $x^{0}$ of $A$ and any $\epsilon>0$, there is a $\delta>0$ such that if $x$ and $x^{\prime}$ are any two points of $A$ with $r_{x x^{\circ}}<\delta$ and $r_{x^{\prime} x^{\circ}}<\delta$, then

$$
\left|R_{k}\left(x^{\prime} ; x\right)\right| \leqq r_{x x^{\prime}}^{m-\sigma_{k}} \epsilon \text {. }
$$

One might define the derivatives of a function at the points of a set $B$, when the function is defined in a larger set $A$. We shall not do this here.

If $m=0,(3.1)$ and (3.2) state merely that $f(x)$ is continuous. Note that the conditions are satisfied automatically at all isolated points of $A$, no matter how the $f_{k}(x)$ are defined there. 
It is easily seen that the $f_{k}(x)$ are continuous in a neighborhood of each point of $A$, and are thus bounded there. From this we prove that if $f(x)$ is of class $C^{m}$ in $A$ in terms of the $f_{k}(x)\left(\sigma_{k} \leqq m\right)$, then it is of class $C^{m^{\prime}}$ in $A\left(m^{\prime}<m\right)$ in terms of the $f_{k}(x)\left(\sigma_{k} \leqq m^{\prime}\right)$.

Any function we shall say is of class $C^{-1}$ in $A . f(x)$ is of class $C^{\infty}$ in $A$ in terms of the $f_{k}(x)$ (defined for all $k$ ) if it is of class $C^{m}$ in $A$ in terms of the $f_{k}(x)\left(\sigma_{k} \leqq m\right)$ for each $m$.

Suppose $f(x)$ is defined throughout the region $R$, and is of class $C^{m}$ in terms of the $f_{k}(x)\left(\sigma_{k} \leqq m\right)$. Then putting $x=\left(x_{1}, \cdots, x_{n}\right), x^{\prime}=\left(x_{1}, \cdots\right.$, $\left.x_{h}+\Delta x_{h}, \cdots, x_{n}\right),(3.1)$ gives

$$
\begin{gathered}
f_{k_{1} \cdots k_{n}}\left(x^{\prime}\right)=f_{k_{1} \cdots k_{n}}(x)+f_{k_{1}, \cdots, k_{h}+1, \cdots, k_{n}}(x) \Delta x_{h} \\
+R_{k_{1} \cdots k_{n}}^{(h)}\left(x^{\prime} ; x\right)
\end{gathered}
$$

(provided $\sigma_{k}<m$ ), where $R_{k_{1}}^{(h)} \ldots k_{n}\left(x^{\prime} ; x\right) / \Delta h \rightarrow 0$ as $\Delta h \rightarrow 0$, which shows that

$$
\frac{\partial}{\partial x_{h}} f_{k_{1} \cdots k_{n}}(x)=f_{k_{1}, \cdots, k_{h}+1, \cdots, k_{n}}(x) \quad\left(\sigma_{k}<m\right)
$$

in $R$; thus in this case, $f(x)$ is of class $C^{m}$ in the ordinary sense, and the $f_{k}(x)$ are the partial derivatives of $f(x)$. The converse is true, by Taylor's Theorem.

4. The main theorem of the present paper is the following:

Theorem I. Let $A$ be a closed subset of $E$, and let $f(x)=f_{0}(x)$ be of class $C^{m}$ ( $m$ finite or infinite) in $A$ in terms of the $f_{k}(x)\left(\sigma_{k} \leqq m\right)$. Then there is a function $F(x)$ of class $C^{m}$ in $E$ in the ordinary sense, such that

(1) $F(x)=f(x)$ in $A$,

(2) $D_{k} F(x)=f_{k}(x)$ in $A\left(\sigma_{k} \leqq m\right)$,

(3) $F(x)$ is analytic in $E-A . \dagger$

Of course (2) includes (1).

No such theorem holds if we leave out the uniformity condition on $R_{k}\left(x^{\prime} ; x\right)$, i.e. if we assume merely that for any $x$ and $\epsilon>0$ there is a $\delta>0$ such that if $r_{x x^{\prime}}<\delta$, then $\left|R_{k}\left(x^{\prime} ; x\right)\right|<r_{x x^{\prime}}^{m-\sigma_{k}} \epsilon$. The following example shows this. Let $A$ be the set of points (using one variable) $x=0,1 / 2^{s}$ and $1 / 2^{s}+1 / 2^{2 s}$ $(s=1,2, \cdots)$. Set $f(x)=0$ at $x=0$ and $1 / 2^{s}$, and $f(x)=1 / 2^{2 s}$ at the remaining points. Set $f_{1}(x) \equiv 0$ in $A$. The above condition is satisfied, but there is no extension of $f(x)$ which has a continuous first derivative.

5. The following lemma will be needed; its proof is elementary.

$\dagger$ It is seen from the proof in $\$ 16$ that $F(x)$ is analytic in a complex region with the following property. If $x$ is a point of $E-A$ distant $3 \rho$ from $A$, then the region contains all points within a distance $\rho$ of $x$. 
LEMMA 1. Let w(z) be a continuous function of one variable defined throughout an interval containing $z_{0}$, let $A^{*}$ be a closed set in this interval, and let $w_{0}^{\prime}$ be a fixed number. Suppose that for every $\epsilon>0$ there is $a \delta>0$ such that

(1) if $z$ is in $A^{*}$ and $\left|z-z_{0}\right|<\delta$, then

$$
\left|w(z)-w\left(z_{0}\right) /\left(z-z_{0}\right)-w_{0}^{\prime}\right|<\epsilon ;
$$

(2) if $z$ is not in $A^{*}$ and $\left|z-z_{0}\right|<\delta$, then the derivative $w^{\prime}(z)$ exists and

$$
\left|w^{\prime}(z)-w_{0}^{\prime}\right|<\epsilon .
$$

Then $w(z)$ has a derivative at $z_{0}$, and $w^{\prime}\left(z_{0}\right)=w_{0}^{\prime}$.

\section{Differentiable extensions}

6. The functions $\psi_{k}\left(x^{\prime} ; x\right)$. We shall make use of functions defined as follows for $x$ in $A$ and $x^{\prime}$ in $E$ ( $m$ finite):

$$
\psi_{k}\left(x^{\prime} ; x\right)=\sum_{\sigma_{l} \leqq m-\sigma_{k}} \frac{f_{k+l}(x)}{l !}\left(x^{\prime}-x\right)^{l} \quad\left(\sigma_{k} \leqq m\right) ;
$$

$\psi_{k}\left(x^{\prime} ; x\right)$ is the value at $x^{\prime}$ of the polynomial of degree at most $m-\sigma_{k}$ which approximates the function $f_{k}(x)$ to the $\left(m-\sigma_{k}\right)$ th order at $x$. Keeping $x$ fixed, it is a polynomial in $x^{\prime}$, given by Taylor's formula in terms of its value and derivatives at $x$. In terms of these functions, (3.1) becomes

$$
f_{k}\left(x^{\prime}\right)=\psi_{k}\left(x^{\prime} ; x\right)+R_{k}\left(x^{\prime} ; x\right) \quad\left(\sigma_{k} \leqq m\right) .
$$

The $l$ th derivative of the function of $x^{\prime} \psi_{k}\left(x^{\prime} ; x\right)$ at $x^{\prime}$ is $\psi_{k+l}\left(x^{\prime} ; x\right)$; if we express $\psi_{k}\left(x^{\prime \prime} ; x\right)$ by Taylor's formula in terms of its value and derivatives at $x^{\prime}$, we obtain

$$
\begin{aligned}
\psi_{k}\left(x^{\prime \prime} ; x\right) & =\sum_{l} \frac{\psi_{k+l}\left(x^{\prime} ; x\right)}{l !}\left(x^{\prime \prime}-x^{\prime}\right)^{l} \\
& =\sum_{l} \frac{\left(x^{\prime \prime}-x^{\prime}\right)^{l}}{l !} \sum_{j} \frac{f_{k+l+j}(x)}{j !}\left(x^{\prime}-x\right)^{j} .
\end{aligned}
$$

The definition of $\psi_{k}\left(x^{\prime \prime} ; x^{\prime}\right)$ in conjunction with this identity gives, for any points $x$ and $x^{\prime}$ in $A$ and $x^{\prime \prime}$ in $E$,

$$
\begin{aligned}
\psi_{k}\left(x^{\prime \prime} ; x^{\prime}\right) & =\sum_{l} \frac{f_{k+l}\left(x^{\prime}\right)}{l !}\left(x^{\prime \prime}-x^{\prime}\right)^{l} \\
& =\sum_{l} \frac{\left(x^{\prime \prime}-x^{\prime}\right)^{l}}{l !}\left[\sum_{j} \frac{f_{k+l+j}(x)}{j !}\left(x^{\prime}-x\right)^{i}+R_{k+l}\left(x^{\prime} ; x\right)\right] \\
& =\psi_{k}\left(x^{\prime \prime} ; x\right)+\sum_{l} \frac{R_{k+l}\left(x^{\prime} ; x\right)}{l !}\left(x^{\prime \prime}-x^{\prime}\right)^{l} .
\end{aligned}
$$


7. The function $\Theta(x)$. Let $R$ be the region given by the inequalities $\left|x_{h}\right|<1(h=1, \cdots, n)$, let $R^{\prime}$ be $R$ minus the origin, and let $R^{*}$ be the boundary of $R$. Define the functions $\theta, \theta^{\prime}, \Theta$ as follows:

$$
\theta(x)=2\left(1-x_{1}^{2}\right) \cdots\left(1-x_{n}^{2}\right)-1 \text { in } R^{\prime},
$$

$$
\theta^{\prime}(x)=\frac{\theta(x)}{1-\theta^{2}(x)} \quad \text { in } R^{\prime}
$$

$$
\Theta(x)=\left\{\begin{array}{l}
e^{\theta^{\prime}(x)} \\
0
\end{array}\right.
$$

in $R^{\prime}$,

in $E-R$.

It is seen that $-1<\theta(x)<+1, \theta(x) \rightarrow+1$ as $x \rightarrow 0$, and $\theta(x) \rightarrow-1$ as $x \rightarrow R^{*}$; hence $\theta^{\prime}(x) \rightarrow+\infty$ as $x \rightarrow 0$ and $\theta^{\prime}(x) \rightarrow-\infty$ as $x \rightarrow R^{*}$. Consequently $\Theta(x) \rightarrow+\infty$ to infinite order as $x \rightarrow 0$ and $\Theta(x) \rightarrow 0$ to infinite order as $x \rightarrow R^{*}$; also $\Theta(x)$ is of class $C^{\infty}$ for $x \neq 0$. If $\Theta^{\prime}(x)=1 / \Theta(x)$ in $R^{\prime}$ and $\Theta^{\prime}(x)=0$ for $x=0$, then $\Theta^{\prime}(x)$ is of class $C^{\infty}$ in $R$.

8. The subdivision of $E-A$. Divide $E$ into $n$-cubes of side 1 , and let $K_{0}$ be the set of all these cubes whose distances from $A$ are at least $6 n^{1 / 2}$ (if there are any). In general, having constructed the cubes of $K_{s-1}$, divide each cube which is now present but is not in $K_{0}+\cdots+K_{\bullet-1}$ into $2^{n}$ cubes of side $1 / 2^{s}$, and let $K_{s}$ be the set of all these cubes whose distances from $A$ are at least $6 n^{1 / 2} / 2^{\circ}$ (if there are any).

The distance from any cube $C$ of $K_{8}$ to $A$ is $\left\langle 18 n^{1 / 2} / 2^{*}(s \geqq 1)\right.$; for it lies in a cube $C^{\prime}$ of the previous subdivision which does not belong to $K_{\bullet-1}$, and whose distance from $A$ is therefore $<6 n^{1 / 2} / 2^{s-1}$.

Any cube $C$ of $K_{s}$ is separated from any cube $C^{\prime}$ of $K_{s+2}$ by at least four cubes of $K_{s+1}$. For the distance from $C$ to $A$ is $\geqq 12 n^{1 / 2} / 2^{s+1}$, the distance from any point of $C^{\prime}$ to $A$ is $<9 n^{1 / 2} / 2^{s+1}$, and the diameter of any cube of $K_{\bullet+1}$ is $n^{1 / 2} / 2^{s+1}$.

9. The functions $\phi_{\nu}(x)$. We introduce the following definitions:

$y^{1}, y^{2}, \cdots$ is the set of all vertices of cubes of $K_{0}+K_{1}+\cdots$, arranged in a sequence.

$r_{\nu}$ is the distance from $y_{\nu}$ to $A(\nu=1,2, \cdots)$.

$x^{*}$ is a fixed point of $A$ whose distance from $y^{\nu}$ is $r_{v}$.

$b$, is the length of side of the largest cube of $K_{0}+K_{1}+\cdots$ with $y^{\nu}$ as a vertex.

$I_{\nu}$ is the set of points $x$ for which $\left|x_{h}-y_{h}{ }^{\nu}\right| \leqq b_{\nu}(h=1, \cdots, n) ; B_{\nu}$ is its boundary. 


$$
\begin{aligned}
\pi_{\nu}(x) & =\Theta\left(\frac{x_{1}-y_{1}{ }^{\nu}}{b_{\nu}}, \ldots, \frac{x_{n}-y_{n}{ }^{\nu}}{b_{\nu}}\right) \text { in } E-y^{\nu} ; \\
\pi_{\nu}^{\prime}(x) & =\Theta^{\prime}\left(\frac{x_{1}-y_{1}{ }^{\nu}}{b_{\nu}}, \ldots, \frac{x_{n}-y_{n}{ }^{\nu}}{b_{\nu}}\right) \text { in } I_{\nu}-B_{\nu} . \\
\phi_{\nu}(x) & =\left\{\begin{array}{crr}
\frac{\pi_{\nu}(x)}{\sum_{\lambda} \pi_{\lambda}(x)} & \text { in } & E-A, x \neq y^{1}, y^{2}, \cdots, \\
1, & x & =y^{\nu}, \\
0, & x & =y^{\mu}(\mu \neq \nu) .
\end{array}\right.
\end{aligned}
$$

Suppose $y^{*}$ is a given point of $E-A$, distant $\delta_{*}$ from $A$ (or from a given point $x^{0}$ of $A$ ), and suppose $y^{*}$ lies in the cube $C$ of $K_{8}$. Then if $I_{\nu}$, with center $y^{\nu}$, has points in common with $C$, and $y^{\nu}$ is distant $d_{\nu}$ from $A$ (or from $x^{0}$ ),

$$
\delta_{*} / 2 \leqq d_{\nu}<2 \delta_{*} .
$$

To prove this, say $C^{\prime}$ is a largest cube with $y^{\prime}$ as a vertex, and $C^{\prime}$ is in $K_{t}$; then $t \geqq s-1$. The diameter of $C^{\prime}$ is $n^{1 / 2} / 2^{t}$; hence $y^{y}$ is distant at most $n^{1 / 2} / 2^{t}$ $\leqq 2 n^{1 / 2} / 2^{s}$ from any point of $I_{\nu}$. As the diameter of $C$ is $n^{1 / 2} / 2^{s}, y^{y}$ is distant at most $3 n^{1 / 2} / 2^{\text {s }}$ from $y^{*}$. But $\delta * \geqq 6 n^{1 / 2} / 2^{s}$, and the inequalities follow.

Each function $\pi_{\nu}(x)$ is $>0$ in $I_{\nu}-B_{\nu}-y^{\nu}$ and only there; it approaches $\infty$ and 0 to infinite order as $x$ approaches $y^{\nu}$ and $B_{\nu}$ respectively. Each point $x$ of $E-A$ is interior to some cube $I_{\nu}$, hence $\pi_{\nu}(x)>0$ for some $\nu$, and $\sum \pi_{\lambda}(x)>0$ in $E-A$, justifying the definition of $\phi_{\nu}(x)$. Note that $\phi_{\nu}(x)$ is $\neq 0$ in $I_{\nu}-B_{\nu}$ and only there; also

$$
\sum_{\nu} \phi_{\nu}(x)=1 \text { in } E-A .
$$

We shall show that $\phi_{\nu}(x)$ is of class $C^{\infty}$ in $E-A$. This is obvious at points $x \neq y^{\nu}$. Consider a small neighborhood $U_{\lambda}$ of $y^{\lambda}, \lambda \neq \nu . \pi_{\lambda}^{\prime}(x)$ is of class $C^{\infty}$ in $U_{\lambda}$; hence the same is true of $\phi_{\nu}=\pi_{\lambda}^{\prime} \pi_{\nu} /\left(1+\pi_{\lambda}^{\prime} \sum_{\mu \neq \lambda} \pi_{\mu}\right)$ in $U_{\lambda}$. Similarly $\phi_{\nu}=1 /\left(1+\pi_{\nu}^{\prime} \sum_{\mu \neq \nu} \pi_{\mu}\right)$ is of class $C^{\infty}$ in a small neighborhood $U_{\nu}$ of $y^{\nu}$; the statement follows.

10. The derivatives of the $\phi_{\nu}(x)$. Consider two (closed) cubes $C$ and $C^{\prime}$ of $K_{0}+K_{1}+\cdots$, and let $J$ and $J^{\prime}$ be those sets $I_{\nu}$ with points in $C$ and $C^{\prime}$ respectively. We shall say $C$ and $C^{\prime}$ are of the same type if the sets in $J^{\prime}$ can be brought into coincidence with the sets in $J$ by a translation and stretching of the axes, that is, if the structure of the subdivision about $C^{\prime}$ is the same as that about $C$. There are but a finite number, say $d$, of possible types of cubes, and for some number $c$, there are at most $c$ sets $I_{\nu}$ with points in any given cube $C$. 
Take a fixed cube $C$ of $K_{0}$ and a fixed $k$. As each $\phi_{\nu}(x)$ is of class $C^{\infty}$, $D_{k} \phi_{\nu}(x)$ is bounded in $C$; there are only a finite number of these functions $\neq 0$ in $C$, and hence they are uniformly bounded:

$$
\left|D_{k} \phi_{\nu}(x)\right|<N_{k}(C) \text { in } C \quad(\nu=1,2, \cdots) .
$$

Consider now any cube $C^{\prime}$ of any $K_{s}$, and let $C$ be a (perhaps hypothetical) cube of $K_{0}$ of the same type as $C^{\prime}$. If $I_{\lambda_{1}}, \cdots, I_{\lambda_{\ell^{\prime}}}$ are the sets $I_{\lambda}$ with points in $C^{\prime}$, let $I_{\lambda_{1}}, \cdots, I_{\lambda_{t}}$ be the corresponding sets with points in $C$; the latter set of sets is carried into the former by a translation of the axes and a stretching by a factor $1 / 2^{s}$. Each function $\phi_{\lambda_{q}}$ corresponding to $I_{\lambda_{q}}$ goes thereby into the function

$$
\phi_{\lambda_{q^{\prime}}}(x)=\phi_{\lambda_{q}}\left[y^{\lambda_{a}}+2^{*}\left(x-y_{q^{\prime}} a^{\prime}\right)\right]
$$

corresponding to $I_{\lambda_{g^{\prime}}}$. Therefore, differentiating $\sigma_{k}$ times with respect to $x$,

$$
D_{k} \phi_{\lambda_{q^{\prime}}}(x)=2^{s \sigma_{k}} D_{k} \phi_{\lambda_{q}}\left[y^{\lambda_{q}}+2^{\bullet}\left(x-y^{\lambda_{q}}{ }^{\prime}\right)\right]
$$

for $x$ in $C^{\prime}$, and hence

$$
\left|D_{k} \phi_{\nu}(x)\right|<2^{{ }^{g \sigma_{k}} N_{k}(C)} \text { in } C^{\prime} \quad(\nu=1,2, \cdots),
$$

as $\phi_{\nu}(x)=0$ in $C^{\prime}$ for $\nu \neq \lambda_{1}^{\prime}, \cdots, \lambda_{t}^{\prime}$. Now the constants $N_{k}(C)$ take on at most $d$ distinct values for a fixed $k$; if we let $N_{k}$ be the largest of these, we can state: Given any $n$-fold set of numbers $k$, there is a number $N_{k}$ such that if $C$ is any cube of $K_{s}$, then

$$
\left|D_{k} \phi_{\nu}(x)\right|<2^{s \sigma_{k}} N_{k} \text { in } C \quad(\nu=1,2, \cdots) .
$$

11. A differentiable extension of $f(x), m$ finite. We are now in a position to prove, for $m$ finite,

LEMMA 2. Under the conditions of Theorem $\mathrm{I}$, there is a function $g(x)$ of class $C^{\infty}$ in $E-A$, having the properties (1) and (2) of Theorem I.

For each $\nu(\nu=1,2, \cdots)$ there are functions $\phi_{\nu}(x)$ and $\psi\left(x ; x^{\nu}\right)=\psi_{0}\left(x ; x^{\nu}\right)$; we put

$$
g(x)= \begin{cases}\sum_{\nu} \phi_{\nu}(x) \psi\left(x ; x^{\nu}\right) & \text { in } E-A, \\ f(x) & \text { in } A .\end{cases}
$$

As the $\phi_{\nu}(x)$ and $\psi\left(x ; x^{\nu}\right)$ are of class $C^{\infty}$ in $E-A$, the same is true of $g(x)$. The function $g(x)=f(x)$ is of class $C^{m}$ at all inner points of $A$, by $\$ 3$. It remains to show that $D_{k} g(x)$ exists, equals $f_{k}(x)$, and is continuous, at all boundary points of $A$, for $\sigma_{k} \leqq m$. 
Take a fixed boundary point $x^{0}$ of $A$, and any $\epsilon, 0<\epsilon<1$. Take

$$
\eta<\epsilon /\left\{2 c[(m+2) !]^{n}\left(108 n^{1 / 2}\right)^{m} N\right\} \text { and } \eta<\epsilon / 6,
$$

where $N$ is the largest of the numbers $N_{k}$ for $\sigma_{k} \leqq m$. Take $M>\left|f_{k}(x)\right|$ $\left(\sigma_{k} \leqq m, x\right.$ in $A$ and $r_{x x 0} \leqq 1$ ), and take

$$
\delta<\epsilon /\left\{6(m+1)^{n} M\right\} \text { and } \delta<1
$$

so small that (3.2) holds at the point $x^{0}$ with $\epsilon$ replaced by $\eta$. Take now any point $y^{*}$ of $E-A$ within a distance $\delta / 4$ of $x^{0}$; we shall show that

$$
\left|D_{k} g\left(y^{*}\right)-f_{k}\left(x^{0}\right)\right|<\epsilon \quad\left(\sigma_{k} \leqq m\right) .
$$

Say the distance from $y^{*}$ to $A$ is $\delta_{*} / 4$ (then $\delta_{*}<\delta$ ), and let $x^{*}$ be a point of $A$ distant $\delta_{*} / 4$ from $y^{*}$. Consider the sum in (6.1) with $x^{\prime}$ and $x$ replaced by $x^{*}$ and $x^{0}$ respectively; as each $l_{h}$ is $\leqq m$, it contains at most $(m+1)^{n}$ terms. If we take the term with $l_{1}=\cdots=l_{n}=0$ to the other side, there is in each remaining term a factor $\left(x_{h}{ }^{*}-x_{h}{ }^{0}\right)^{l_{h}}$ with $l_{h}>0$. As each $\left|x_{h}{ }^{*}-x_{h}{ }^{0}\right|$ is $<\delta<1$, we find

$$
\left|\psi_{k}\left(x^{*} ; x^{0}\right)-f_{k}\left(x^{0}\right)\right|<(m+1)^{n} M \delta<\epsilon / 6 .
$$

But also $\left|R_{k}\left(x^{*} ; x^{0}\right)\right|<\eta<\epsilon / 6$; hence, using (6.2),

$$
\left|f_{k}\left(x^{*}\right)-f_{k}\left(x^{0}\right)\right|<\epsilon / 3 \text {. }
$$

Similarly we see that $\left|\psi_{k}\left(y^{*} ; x^{*}\right)-f_{k}\left(x^{*}\right)\right|<\epsilon / 6$; therefore

$$
\left|\psi_{k}\left(y^{*} ; x^{*}\right)-f_{k}\left(x^{0}\right)\right|<\epsilon / 2 \quad\left(\sigma_{k} \leqq m\right) .
$$

Say $y^{*}$ lies in the cube $C$ of $K_{s}$, and let $I_{\lambda_{1}}, \cdots, I_{\lambda_{t}}$ be those sets $I_{\lambda}$ with points in $C$. Each corresponding point $y^{\lambda} q$ is distant $<\delta / 2$ from $x^{0}$, by (9.1), and hence each corresponding point $x^{\lambda} \varepsilon$ is distant $<\delta$ from $x^{0}$. As the same is true of $x^{*},(3.2)$ gives

$$
\left|R_{k}\left(x^{y} ; x^{*}\right)\right| \leqq r_{x^{y} x^{*}}^{m-\sigma_{k}} \eta \quad \quad\left(\nu=\lambda_{1}, \cdots, \lambda_{t}\right)
$$

Set

$$
\zeta_{\nu ; k}(x)=\psi_{k}\left(x ; x^{\nu}\right)-\psi_{k}\left(x ; x^{*}\right) \quad\left(\nu=\lambda_{1}, \cdots, \lambda_{t}\right) ;
$$

then as $r_{x^{\nu} x^{*}}<\delta_{*}$ and $\left|x_{h}-x_{h}{ }^{\nu}\right|<\delta_{*}$ for $x$ in $C,\left|\left(x-x^{\nu}\right)^{l}\right|<\delta_{*}^{\sigma_{l}}$, and (6.3) and (11.4) give

$$
\left|\zeta_{\nu ; k}(x)\right|<(m+1)^{n} \delta_{*}{ }^{m-\sigma_{k} \eta} \text { in } C \quad\left(\nu=\lambda_{1}, \cdots, \lambda_{t}\right) .
$$

Using (9.2), we see that

$$
g(x)=\psi\left(x ; x^{*}\right)+\sum_{s=1}^{t} \phi_{\lambda_{0}}(x) \zeta_{\lambda_{s} ; 0}(x) \text { in } C .
$$


As $D_{k} \psi\left(x ; x^{\nu}\right)=\psi_{k}\left(x ; x^{\nu}\right)$ and therefore $D_{k} \zeta_{\nu ; 0}(x)=\zeta_{\nu ; k}(x)$,

$$
D_{k} g(x)=\psi_{k}\left(x ; x^{*}\right)+\sum_{s=1}^{t} \sum_{l}\left(\begin{array}{l}
k \\
l
\end{array}\right) D_{l} \phi_{\lambda_{e}}(x) \zeta_{\lambda_{\imath} ; k-l}(x) \text { in } C .
$$

(10.1) and (11.5) give, as $t \leqq c($ see $\S 10) \cdot$ and

$$
\left(\begin{array}{c}
k_{h} \\
l_{h}
\end{array}\right) \leqq m !
$$

$$
\left|D_{k} g(x)-\psi_{k}\left(x ; x^{*}\right)\right|<\sum_{l} c[(m+1) !]^{n} 2^{s \sigma_{l}} N \delta_{*}{ }^{m-\sigma_{k}+\sigma_{l} \eta} \text { in } C .
$$

Now the distance from $C$ to $A$ is $>\delta_{*} / 6$; also, as $C$ is in $K_{s}$, this distance is $<18 n^{1 / 2} / 2^{s}$. Hence $18 n^{1 / 2} / 2^{*}>\delta_{*} / 6$, or, $2^{s}<108 n^{1 / 2} / \delta_{*}$. This gives, as $\sigma_{k} \leqq m$ and $\delta *<1$,

$$
\left|D_{k} g(x)-\psi_{k}\left(x ; x^{*}\right)\right|<c[(m+2) !]^{n}\left(108 n^{1 / 2}\right)^{m} N \delta_{*}{ }^{m-\sigma_{k}} \eta<\epsilon / 2
$$

in $C$, and in particular, at $y^{*}$. This inequality together with (11.3) gives (11.2), as required.

The proof can now be completed with the aid of Lemma 1. (11.2) with $k=0$ shows that $g(x)$ is continuous throughout $E$. Take any number $k=\left(k_{1}, \cdots, k_{n}\right)$ with $\sigma_{k}<m$, and put $k^{\prime}=\left(k_{1}, \cdots, k_{h}+1, \cdots, k_{n}\right)$. Assuming that $D_{k} g(x)$ is continuous in $E$, we shall show that $D_{k^{\prime}} g(x)$ exists and is continuous in $E$. Take any boundary point $x^{0}=\left(x_{1}{ }^{0}, \cdots, x_{n}{ }^{0}\right)$ and put $z_{0}=x_{h}{ }^{0}, w(z)=w\left(x_{h}\right)=D_{k} g\left(x_{1}{ }^{0}, \cdots, x_{h}, \cdots, x_{n}{ }^{0}\right), w_{0}^{\prime}=f_{k^{\prime}}\left(x^{0}\right)$. Let $A^{*}$ be the set of points of $A$ for which $x_{p}=x_{p}{ }^{0}(p \neq h)$. (3.3) with $x=x^{0}$ and $\Delta x_{h}$ $=x_{h}-x_{h}{ }^{0}$, and (11.2) with $k$ replaced by $k^{\prime}$, show that the conditions of the lemma are fulfilled; hence $\partial w\left(z_{0}\right) / \partial x_{h}=D_{k^{\prime}} g\left(x^{0}\right)$ exists and equals $f_{k^{\prime}}\left(x^{0}\right)$. (11.2) shows that $D_{k^{\prime}} g\left(x^{0}\right)$ is continuous at $x^{0}$. Therefore $g(x)$ is of class $C^{m}$ in $E$.

12. A differentiable extension of $f(x), m$ infinite. We now prove Lemma 2 for the case $m=\infty$. For any given $m$, let $\psi_{m ; k}\left(x^{\prime} ; x\right)\left(\sigma_{k} \leqq m\right)$ be the function given by the right hand side of (6.1). Choose the axes so that the origin falls on a point of $A$. Let $S_{p}$ be the set of all points of $E$ whose distances from the origin are $\leqq 2^{p}, p=1,2, \cdots$. Let $M_{p}$ be the maximum of $\left|f_{k}(x)\right|$ for $\sigma_{k} \leqq p$ and $x$ in $A \cdot S_{p}$, and let $N^{(p)}$ be the maximum of $N_{k}$ for $\sigma_{k} \leqq p$. Choose for each positive integer $p$ a number $\delta_{p}$ such that

$$
\delta_{p}<1 /\left\{2^{2 p+1} c[(p+2) !]^{n}\left(36 n^{1 / 2}\right)^{p} N^{(p)} M_{p+1}\right\}, \delta_{p}<\delta_{p-1} / 2 .
$$

The extension $g^{(\infty)}(x)$ of $f(x)$ is determined as follows. Given any number $\nu$, determine the number $\gamma_{\nu}$ so that $\delta_{\gamma_{\nu}+1} \leqq r_{\nu}<\delta_{\gamma_{\nu}}$ (see $\left.\S 9\right)$; set $\gamma_{\nu}=0$ if $r_{\nu}>\delta_{1}$. 
Put

$$
g^{(\infty)}(x)= \begin{cases}\sum_{\nu} \phi_{\nu}(x) \psi_{\gamma_{\nu} ; 0}\left(x ; x^{\nu}\right) & \text { in } E-A, \\ f(x) & \text { in } A .\end{cases}
$$

Given any fixed $k$, we shall find an inequality similar to (11.2) for $D_{k} g^{(\infty)}(x)$. Let $g^{(m)}(x)$ be the extension of $f(x)$ of class $C^{m}$ given by Lemma 2 $(m=1,2, \cdots)$. Given any boundary point $x^{0}$ of $A$ and any $\epsilon>0$, choose $p \geqq \sigma_{k}+2$ so that $x^{0}$ lies in $S_{p}$ and so that $1 / 2^{p}<\epsilon$. Take $\delta<\delta_{p}$ so that (11.2) with $g$ replaced by $g^{\left(\sigma_{k}\right)}$ will hold for our given $k$ and any $y^{*}$ of $E-A$ within $\delta$ of $x^{0}$; we show next that for any such $y^{*}$,

$$
\left|D_{k} g^{(\infty)}\left(y^{*}\right)-D_{k} g^{\left(\sigma_{k}\right)}\left(y^{*}\right)\right|<\epsilon .
$$

Choose $q$ so that $\delta_{q+1} \leqq \delta_{*}<\delta_{q}$, where $\delta_{*}$ is the distance from $y^{*}$ to $A$; then $q \geqq p$. Define $C, K_{s}, I_{\lambda_{1}}, \cdots, I_{\lambda_{t}}$ as in $\$ 11$. Note that for $\nu=$ any $\lambda_{h}, \delta_{\gamma_{\nu}+1}$ $\leqq r_{\nu}<2 \delta<2 \delta_{p}<\delta_{p-1}$, hence $\gamma_{\nu}+1>p-1$, and thus $\gamma_{v}>p-2 \geqq \sigma_{k}$. Set

$$
\xi_{\nu}(x)=\psi_{\gamma_{\nu} ; 0}\left(x ; x^{\nu}\right)-\psi_{\sigma_{k} ; 0}\left(x ; x^{\nu}\right)
$$$$
\left(\nu=\lambda_{1}, \cdots, \lambda_{t}\right) \text {; }
$$

using (12.1) and (11.1), we see that

$$
g^{(\infty)}(x)=g^{\left(\sigma_{k}\right)}(x)+\sum_{u=1}^{t} \phi_{\lambda_{u}}(x) \xi_{\lambda_{u}}(x) \text { in } C .
$$

Now $D_{j} \xi_{\nu}(x)=\psi_{\gamma_{p} ; j}\left(x ; x^{\nu}\right)-\psi_{\sigma_{k} ; j}\left(x ; x^{\nu}\right)$. If we replace $k$ by $j$ in (6.1), then those and only those terms in the sum with $\sigma_{l} \leqq m-\sigma_{j}$ occur. Replacing $m$ by $\gamma_{\nu}$ and $\sigma_{k}$ successively and subtracting, we have

$$
D_{j} \xi_{\nu}(x)=\sum_{\sigma_{l}=\sigma_{k}=\sigma_{j}+1}^{\gamma_{\nu}-\sigma_{j}} \frac{f_{j+l}\left(x^{\nu}\right)}{l !}\left(x-x^{\nu}\right)^{l} \text { in } C .
$$

Now $r_{\nu}>\delta_{*} / 2$, by (9.1), hence $r_{\nu}>\delta_{q+2}$, and thus $\gamma_{\nu} \leqq q+1\left(\nu=\lambda_{1}, \cdots, \lambda_{t}\right)$; there are therefore less than $(q+2)^{n}$ terms in the sum, and in each term, $\sigma_{j}+\sigma_{l} \leqq q+1$. It follows that $\left|f_{j+l}\left(x^{\nu}\right)\right|<M_{q+1}$ in each term. Also $\left|x_{h}-x_{h}{ }^{\prime}\right|$ $<2 \delta_{*}<2 \delta_{q}$ and $\sigma_{l} \geqq \sigma_{k}-\sigma_{j}+1$ in each term; hence

$$
\left|D_{j \xi_{\nu}}(x)\right|<(q+2)^{n} M_{q+1} 2^{q+1} \delta_{*}{ }^{\sigma-\sigma_{j}} \delta_{q}
$$

in $C$. This with (12.4) gives

$$
\begin{gathered}
\left|D_{k} g^{(\infty)}(x)-D_{k} g^{\left(\sigma_{k}\right)}(x)\right| \leqq \sum_{u=1}^{t} \sum_{i}\left(\begin{array}{l}
k \\
j
\end{array}\right)\left|D_{k-j} \phi_{\lambda_{u}}(x)\right|\left|D_{j} \zeta_{\lambda_{u}}(x)\right| \\
<c \sum_{j}\left(\begin{array}{l}
k \\
j
\end{array}\right) 2^{s\left(\sigma_{k}-\sigma_{j}\right)} N^{\left(\sigma_{k}\right)}(q+2)^{n} M_{q+1} 2^{a+1} \delta_{*}{ }^{\sigma k-\sigma_{j}} \delta_{q}
\end{gathered}
$$


in $C$. Now the distance from $C$ to $A$ is $>\delta_{*} / 2$ and is $\left\langle 18 n^{1 / 2} / 2^{*} ;\right.$ hence $2 \cdot<36 n^{1 / 2} / \delta_{*}$. Also $\sigma_{k}<p \leqq q$; therefore

$$
\begin{aligned}
\left|D_{k} g^{(\infty)}(x)-D_{k} g^{\left(\sigma_{k}\right)}(x)\right|<c[(q+2) !]^{n}\left(36 n^{1 / 2}\right)^{q} N^{(q)} M_{q+1} 2^{q+1} \delta_{q} \\
<1 / 2^{q}<\epsilon
\end{aligned}
$$

in $C$, and in particular, at $y^{*}$, proving (12.2). Using (11.2), we find $\left|D_{k} g^{(\infty)}\left(y^{*}\right)-f_{k}\left(x^{0}\right)\right|<2 \epsilon$ for any point $y^{*}$ of $E-A$ within $\delta$ of $x^{0}$. Again we can apply Lemma 1 and show that $D_{k} g^{(\infty)}(x)$ exists and is continuous throughout $E$. As this is true for every $k$, the proof is complete.

13. We prove next a combined extension and approximation theorem.

LEMMa 3. Let $f(x)$ be of class $C^{m}$ ( $m$ finite) in $E$, with $D_{k} f(x)=f_{k}(x)\left(\sigma_{k} \leqq m\right)$ there, and let $f_{k}(x)\left(m<\sigma_{k} \leqq m^{\prime}, m^{\prime}>m\right.$ finite or infinite $)$ be defined in the closed set $A$ so that $f(x)$ (considered now only in $A$ ) is of class $C^{m^{\prime}}$ there. Then for an arbitrary $\epsilon>0$ there is a function $g(x)$ which is of class $C^{m}$ in $E$, of class $C^{m^{\prime}}$ in a neighborhood of $A$, and equals $f(x)$ outside another neighborhood of $A$, such that

$$
D_{k} g(x)=f_{k}(x) \text { in } A \quad\left(\sigma_{k} \leqq m^{\prime}\right) \text {, }
$$

and

$$
\left|D_{k} g(x)-D_{k} f(x)\right|<\epsilon \text { in } E \quad\left(\sigma_{k} \leqq m\right) .
$$

Let $f^{\prime}(x)$ be the extension of class $C^{m^{\prime}}$ of the values of $f(x)$ in $A$ given by the last lemma, and put $\zeta(x)=f^{\prime}(x)-f(x)$; then $\zeta(x)$ is of class $C^{m}$ in $E$, and

$$
D_{k} \zeta(x)=0 \text { in } A
$$$$
\left(\sigma_{k} \leqq m\right) \text {. }
$$

Set $\eta=\epsilon /\left\{c[(m+1) !]^{n}\left(36 n^{1 / 2}\right)^{m} N\right\} \quad\left(N=\max N_{k}\right.$ for $\left.\sigma_{k} \leqq m\right)$. As $\zeta(x)$ is of class $C^{m}$ and $D_{k} \zeta(x)$ vanishes in $A\left(\sigma_{k} \leqq m\right)$, we can find an open set $R$ containing $A$ so that if $y$ is any point of $R-A$, at a distance $\delta$ from $A$, then

$$
\left|D_{k} \zeta(y)\right|<\eta \delta^{m-\sigma_{k}} \quad\left(\sigma_{k} \leqq m\right) .
$$

Let $\nu_{1}, \nu_{2}, \cdots$ be those numbers such that $I_{\nu_{p}}$ lies wholly in $R(p=1,2, \cdots)$. We set

$$
g(x)=f(x)+\zeta(x) \sum_{p=1}^{\infty} \phi_{v_{p}}(x) \text { in } E-A,
$$

and $g(x)=f(x)$ in $A$. As $\sum \phi_{\nu_{p}}(x)=1$ in an open set surrounding $A, g(x)=f^{\prime}(x)$ there. As $\sum \phi_{\nu_{p}}(x)=0$ in $E-R, g(x)=f(x)$ there. The statements about the class of $g(x)$ are true. To show that (13.2) holds, let $y$ be a point of $R-A$, distant $\delta$ from $A$; then, defining $C, K_{s}, I_{\lambda_{1}}, \cdots, I_{\lambda_{t}}$ as in the previous lemma, we have 


$$
\begin{aligned}
\left|D_{k} g(y)-D_{k} f(y)\right| & \leqq \sum_{p} \sum_{l}\left(\begin{array}{l}
k \\
l
\end{array}\right)\left|D_{l} \phi_{\nu_{p}}(y)\right|\left|D_{k-l} \zeta(y)\right| \\
& <c \sum_{l}(m !)^{n} 2^{s \sigma_{l}} N \delta^{m-\sigma_{k}+\sigma_{l} \eta}<\epsilon \quad\left(\sigma_{k} \leqq m\right) .
\end{aligned}
$$

14. We close this section with a theorem concerning the isolated points of $A$. Define $\alpha_{p}$ as follows:

$$
\alpha_{p}=\left\{\begin{array}{l}
m \text { if } m \text { is finite } \\
p \text { if } m \text { is infinite } \quad(p=1,2, \cdots) .
\end{array}\right.
$$

Lemma 4. Consider the closed set $A=A^{\prime}+a_{1}+a_{2}+\cdots$, where $a_{1}, a_{2}, \ldots$ are isolated points (then $A^{\prime}$ is closed), and let $m$ be finite or infinite. Let $f_{k}(x)$ be defined in $A^{\prime}$ for $\sigma_{k} \leqq m$ and at each $a_{s}$ for all $k$, so that $f(x)$ is of class $C^{m}$ in $A$ in terms of the $f_{k}(x)\left(\sigma_{k} \leqq m\right)$. Then there is a function $g^{\prime}(x)$ of class $C^{\infty}$ in $E-A^{\prime}$ and of class $C^{m}$ in $E$, such that

$$
D_{k} g^{\prime}(x)=f_{k}(x) \text { in } A^{\prime} \text { for } \sigma_{k} \leqq m \text { and at each } a_{p} \text { for all } k \text {. }
$$

Let $g(x)$ be the extension of $f(x)$ of class $C^{m}$ given by Lemma 2. Let $U_{1}, U_{2}, \cdots$ be neighborhoods of $a_{1}, a_{2}, \cdots$, chosen so that each is at a positive distance from each other and from $A^{\prime}$. If $m$ is finite, we alter $g(x)$ in $U_{1}$, next in $U_{2}$, etc., by means of the last lemmat, so that the new function $g^{\prime}(x)$ will take on the required derivatives at $a_{1}+a_{2}+\cdots$, and so that

$$
\left|D_{k} g^{\prime}(x)-D_{k} g(x)\right|<1 / p \text { in } U_{p}\left(\sigma_{k} \leqq \alpha_{p}, p=1,2, \cdots\right) .
$$

(14.2) is an immediate consequence of this inequality and Lemma 1.

\section{Approximation theorems}

15. We prove first the following extension of the Weierstrass approximation theorem. $\ddagger$

Lemma 5. Let $g(x)$ be of class $C^{m}$ in $E$ ( $m$ finite), and let $S$ be a bounded closed set in $E$. $\$$ Then for each $\epsilon>0$ there exists a function $G(x)$ analytic in $E$ and such that

$$
\left|D_{k} G(x)-D_{k} g(x)\right|<\epsilon \text { in } S \quad\left(\sigma_{k} \leqq m\right) .
$$

Let $R_{b}^{r}$ be the set of points distant at most $b$ from the origin $(b \geqq 0)$. Consider the $n$-tuple integral

$\dagger$ We use the last lemma with $A$ replaced by $a_{\nu}$ and $m^{\prime}$ by $\infty$.

‡ Compare de la Vallee Poussin, Cours d'A nalyse, vol. II, 2d edition, 1912, pp. 126-137. Lemma 2 .

$\S$ It is sufficient that $g(x)$ be defined over $S$, for we can then extend its definition over $E$, by 


$$
\Phi(b)=T \int_{R_{b}} e^{-r_{0 y}^{2}} d y=T \int \cdots \int e^{-\left(y_{1}{ }^{2}+\cdots+y_{n}{ }^{2}\right)} d y_{1} \cdots d y_{n},
$$

where $T$ is chosen so that $\Phi(\infty)=1$; then $0 \leqq \Phi(b) \leqq 1$ for all $b$. If we replace $y$ by $\kappa y$ and $b$ by $k b$, we see that

$$
\Phi(\kappa b)=T \kappa^{n} \int_{R_{b}} e^{-\kappa^{2} r_{0 y}^{2}} d y .
$$

Let $v(x)$ be a function $\equiv 1$ in $S, \equiv 0$ outside some neighborhood of $S$, and of class $C^{\infty}$ in $E$, such that $D_{k} v(x)=0$ in $S$ for all $k$. (Such a function may be found for instance by the aid of Lemma 2.) Put $g^{\prime}(x)=v(x) g(x)$, and

$$
G(x)=T \kappa^{n} \int_{E} g^{\prime}(y) e^{-\kappa^{2} r_{x}^{2}} d y,
$$

where $\kappa$ will be chosen later; $G(x)$ is analytic in $E$. As $r_{x y}$ is a function of $y-x$ alone, differentiating under the integral sign gives

$$
D_{k} G(x)=T \kappa^{n} \int_{E} g^{\prime}(y) D_{k}^{(x)} e^{-\kappa^{2} r_{x y}^{2} d y}=(-1)^{\sigma_{k}} T \kappa^{n} \int_{E} g^{\prime}(y) D_{k}(y) e^{-\kappa^{2} r_{x y}^{2}} d y,
$$

where $D_{k}^{(x)}$ and $D_{k}^{(y)}$ denote differentiation with respect to $x$ and $y$ respectively. Integrating by parts $\sigma_{k}$ times gives

$$
D_{k} G(x)=T \kappa^{n} \int_{E} D_{k} g^{\prime}(y) e^{-\kappa^{2} r_{x y}^{2}} d y .
$$

As $\Phi(\infty)=1$, we see that

$$
D_{k} G(x)-D_{k} g^{\prime}(x)=T \kappa^{n} \int_{E}\left[D_{k} g^{\prime}(y)-D_{k} g^{\prime}(x)\right] e^{-\kappa^{2} r_{x y}^{2}} d y
$$

Take $M$ so large that

$$
\left|D_{k} g^{\prime}(x)\right|<M \text { in } E
$$

$$
\left(\sigma_{k} \leqq m\right) \text {. }
$$

The functions $D_{k} g^{\prime}(x)$ are uniformly continuous in $E$; hence there is a $\delta>0$ such that

$$
\left|D_{k} g^{\prime}(y)-D_{k} g^{\prime}(x)\right|<\epsilon / 2 \quad\left(r_{x y}<\delta, \sigma_{k} \leqq m\right) .
$$

Take $\kappa$ so large that

$$
1-\Phi(\kappa \delta)<\epsilon /(4 M) .
$$

For a given $x$, let $U$ consist of all points within $\delta$ of $x$; then if $J_{1}$ and $J_{2}$ are formed by replacing the domain of integration on the right hand side of 
(15.6) by $U$ and $E-U$ respectively, we have, using (15.3),

$$
\begin{aligned}
& \left|J_{1}\right|<T \kappa^{n} \int_{U} \frac{\epsilon}{2} e^{-\kappa^{2} r_{z y}^{2}} d y=\frac{\epsilon}{2} \Phi(\kappa \delta)<\frac{\epsilon}{2}, \\
& \left|J_{2}\right|<T \kappa^{n} \int_{E-U} 2 M e^{-\kappa^{2} r_{x y}^{2}} d y=2 M[1-\Phi(\kappa \delta)]<\frac{\epsilon}{2},
\end{aligned}
$$

and hence $\left|D_{k} G(x)-D_{k} g^{\prime}(x)\right|<\epsilon$ in $E\left(\sigma_{k} \leqq m\right)$, which gives (15.1).

$G(x)$ may of course be replaced by a polynomial if desired.

16. The above lemma can be generalized as follows.

Lemma 6. Let $R$ be an open set and let $R_{1}, R_{2}, \cdots$ be bounded open sets (some of which may be void) whose sum is $R$, such that each $\bar{R}_{p}=R_{p}$ plus boundary is in $R_{p+1}$. Then if $g(x)$ is defined and of class $C^{m}$ ( $m$ finite or infinite) in $R$, and $\epsilon_{1} \geqq \epsilon_{2} \geqq \cdots$ are given positive numbers, there is an analytic function $G(x)$ defined in $R$ such that

$$
\left|D_{k} G(x)-D_{k} g(x)\right|<\epsilon_{p} \text { in } R-R_{p} \quad\left(\sigma_{k} \leqq \alpha_{p}, p=1,2, \cdots\right) .
$$

$\alpha_{p}$ is defined in (14.1). Note that, if $R_{1}, \cdots, R_{q}$ are void, then

$$
\left|D_{k} G(x)-D_{k} g(x)\right|<\epsilon_{q} \text { in } R \quad\left(\sigma_{k} \leqq \alpha_{q}\right) .
$$

Consider the closed set $\bar{R}_{p-1}+\left(\bar{R}_{p+1}-R_{p}\right)+\left(E-R_{p+2}\right)=Q_{p}^{\prime}+Q_{p}+Q_{p}^{\prime \prime}$; if in Lemma 2 we replace $A$ by this set and $f(x)$ by a function $\equiv 1$ in $Q_{p}$ and $\equiv 0$ in $Q_{p}^{\prime}+Q_{p}^{\prime \prime}$, we find a function $u_{p}(x)$ for each $p$, of class $C^{\infty}$ in $E$, such that

$$
u_{p}(x)=\left\{\begin{array}{l}
1 \text { in } Q_{p}, \\
0 \text { in } Q_{p}^{\prime}+Q_{p}^{\prime \prime} ;
\end{array} \quad D_{k} u_{p}(x)=0 \text { in } Q_{p}^{\prime}+Q_{p}+Q_{p}^{\prime \prime}\left(\sigma_{k}>0\right) .\right.
$$

(If $R_{p+1}$ is void, we put $u_{p}(x)^{\circ} \equiv 0$; if $R_{p+1}$ is not void but $R_{p-1}$ is void, we have $u_{p}(x)=0$ in $Q_{p}^{\prime \prime}$ and $=1$ in $\bar{R}_{p+1}$.) Let $Z_{p} \geqq 1$ be such a number that

$$
\left|D_{k} u_{p}(x)\right|<Z_{p} \text { in } E \quad\left(\sigma_{k} \leqq \alpha_{p}, p=1,2, \cdots\right) .
$$

We define successively analytic functions $G_{1}(x), G_{2}(x), \cdots$, by the following formula:

$$
G_{p}(x)=T_{\kappa_{p}^{n}} \int_{E} u_{p}(y)\left[g(y)-\left\{G_{1}(y)+\cdots+G_{p-1}(y)\right\}\right] e^{-x_{p}^{2} r_{x y}^{2}} d y .
$$

(For $p=1$, the factor in brackets is simply $g(y)$.) $\kappa_{p}$ is chosen so that, if we set

$$
H_{p}(x)=u_{p}(x)\left[g(x)-\left\{G_{1}(x)+\cdots+G_{p-1}(x)\right\}\right],
$$

then 
(16.7)

$$
\begin{array}{r}
\left|D_{k} G_{p}(x)-D_{k} H_{p}(x)\right|<\beta_{p}^{\prime}=\epsilon_{p+1} /\left\{2^{p+2}\left[\left(\alpha_{p+1}+1\right) !\right]^{n} Z_{p+1}\right\} \\
\text { in } \bar{R}_{p+1} \quad\left(\sigma_{k} \leqq \alpha_{p+1}\right)
\end{array}
$$

(see Lemma 5); we shall restrict $\kappa_{p}$ further later. Remembering the definition of $u_{p}(x)$, we see that (16.7) with (16.6) gives

$$
\left|D_{k} g(x)-D_{k}\left\{G_{1}(x)+\cdots+G_{p}(x)\right\}\right|<\beta_{p}^{\prime}<\epsilon_{p} / 2 \text { in } Q_{p}\left(\sigma_{k} \leqq \alpha_{p+1}\right) .
$$

Differentiating $H_{p}(x)$ and using (16.4) and (16.8) with $p$ replaced by $p-1$, we see that (compare the derivation of (11.7))

$$
\left|D_{k} H_{p}(x)\right|<\left[\left(\alpha_{p}+1\right) !\right]^{n} Z_{p} \beta_{p-1}^{\prime}=\epsilon_{p} / 2^{p+1} \text { in } Q_{p-1} \quad\left(\sigma_{k} \leqq \alpha_{p}\right) .
$$

As $u_{p}(x)$ and its derivatives are 0 in $R_{p-1}$, this holds in $R_{p-1}$ also; hence, using (16.7), we have

$$
\left|D_{k} G_{p}(x)\right|<\epsilon_{p} / 2^{p} \text { in } \bar{R}_{p} \quad\left(\sigma_{k} \leqq \alpha_{p}\right) .
$$

We set now

$$
G(x)=G_{1}(x)+G_{2}(x)+\cdots ;
$$

this is the desired approximation to $g(x)$. To prove this, we see first from (16.9) that $D_{k}\left[G_{1}(x)+\cdots+G_{p}(x)\right]$ converges uniformly in any bounded closed subset of $R\left(\sigma_{k} \leqq m\right)$; hence $G(x)$ is defined in $R$, and

$$
D_{k} G(x)=D_{k} G_{1}(x)+D_{k} G_{2}(x)+\cdots \text { in } R \quad\left(\sigma_{k} \leqq m\right) .
$$

Next (16.9) shows that

$$
\begin{aligned}
& \left|D_{k} G_{p+1}(x)+D_{k} G_{p+2}(x)+\cdots\right|<\epsilon_{p+1} / 2^{p+1}+\epsilon_{p+2} / 2^{p+2}+\cdots \\
& \quad \leqq \epsilon_{p}\left(1 / 2^{2}+1 / 2^{3}+\cdots\right)=\epsilon_{p} / 2 \text { in } \bar{R}_{p+1} \quad\left(\sigma_{k} \leqq \alpha_{p+1}\right)
\end{aligned}
$$

this with (16.8) gives $\left|D_{k} G(x)-D_{k} g(x)\right|<\epsilon_{p}$ in $Q_{p}\left(\sigma_{k} \leqq \alpha_{p}\right)$, proving (16.1).

It remains to be shown that $G(x)$ is analytic in $R$. To this end we extend the definition of each $G_{p}(x)$ to complex values of $x=\left(x_{1}^{\prime}+i x_{1}^{\prime \prime}, \cdots\right.$, $\left.x_{n}^{\prime}+i x_{n}{ }^{\prime \prime}\right)$, using (16.5) still. Consider the analytic function of $x$

$$
r_{x y}^{2}=\sum\left(y_{h}-x_{h}\right)^{2}=\sum\left[\left(y_{h}^{\prime}-x_{h}^{\prime}\right)+i\left(y_{h}^{\prime \prime}-x_{h}^{\prime \prime}\right)\right]^{2} ;
$$

as $y_{h}^{\prime \prime}=0$ in (16.5), the domain of integration being real,

$$
\Re\left(r_{x y}^{2}\right)=\sum\left[\left(y_{h}^{\prime}-x_{h}^{\prime}\right)^{2}-x_{h}^{\prime \prime 2}\right] .
$$

Take any point $x^{0}$ of $R$ and let $U$ be the complex region of radius $\bullet \rho$ about $x^{0}$, where $\rho$ is so small that the real points in the complex region of radius $3 \rho$ about $x^{0}$ lie in some $R_{q}$; we take $q$ so that $3 \rho^{2}>1 / 2^{q}$. Now if $p>q, x$ is in $U$, and $y$ is in $R-R_{p-1}$, then $\sum x_{h}^{\prime \prime 2}<\rho^{2}$ and $\sum\left(y_{h}^{\prime}-x_{h}^{\prime}\right)^{2} \geqq 4 \rho^{2}$, and hence

$$
\Re\left(r_{x y}^{2}\right)>3 \rho^{2} \text {. }
$$


Also $H_{p}(y)$ vanishes in $R_{q}$ and in $E-R_{p+2}$ for $p>q$; therefore if $M_{p}^{\prime}$ is the maximum of $\left|H_{p}(y)\right|$ (note that $H_{p}(y)$ is determined before we determine $\left.\kappa_{p}\right)$ and $V_{p}$ is the volume of $R_{p}(p=1,2, \cdots)$,

$$
\begin{aligned}
\left|G_{p}\left(x^{\prime}+i x^{\prime \prime}\right)\right| & <T \kappa_{p}^{n} \int_{R_{p+2}-R_{p-1}} M_{p}^{\prime} e^{-3 \kappa_{p}^{2} \rho^{2}} d y \\
& <T \kappa_{p}^{n} e^{-\kappa_{p}^{2} / 2^{p}} M_{p}^{\prime} V_{p+2}
\end{aligned}
$$

for $x$ in $U$ and $p>q$. Hence if we choose $\kappa_{p}$ successively for $p=1,2, \cdots$, so that this quantity is $<1 / 2^{p}$ (and so that (16.7) holds), then the series in (16.10), when defined for complex values of $x$, converges uniformly in a complex neighborhood of any point of $R$. Therefore the function $G(x)$ is analytic in $R$, completing the proof.

17. The numbers $\kappa_{p}$ as chosen above depend not only on the functions $u_{p}(x)$ but also on the function $g(x)$. Under certain restrictions, we can take them independent of $g(x)$, as follows.

LEMma 7. Let the open sets $R_{1}, R_{2}, \cdots$, the numbers $\epsilon_{1}, \epsilon_{2}, \cdots$, and the functions $u_{1}(x), u_{2}(x), \cdots$ be given as in Lemma 6 ; let $\Delta_{1}(r), \Delta_{2}(r), \cdots$ be a sequence of positive continuous functions defined for $r>0$, such that $\Delta_{p}(r) \rightarrow 0$ as $r \rightarrow 0$ and $\Delta_{p+1}(r) \geqq \Delta_{p}(r)$; let a be a point of $R$, and $M$ a positive number. Then there is a sequence of numbers $\kappa_{1}, \kappa_{2}, \cdots$, with the following property. If $g(x)$ is any function of class $C^{m}$ defined in $R$ such that $|g(a)| \leqq M$ and

$$
\left|D_{k} g\left(x^{\prime}\right)-D_{k} g(x)\right|<\Delta_{p}\left(r_{x x^{\prime}}\right) \text { in } \bar{R}_{p} \quad\left(\sigma_{k} \leqq \alpha_{p}, p=1,2, \cdots\right),
$$

and if $G(x)$ is defined in terms of $g(x)$ as in the previous lemma, using the above numbers $\kappa_{p}$, then $G(x)$ is analytic in $R$ and (16.1) holds.

As the $u$ 's and their derivatives are uniformly continuous in $E$, there are functions $\Gamma_{p}(x)$ of the same sort as the $\Delta$ 's above such that

$$
\left|D_{k} u_{p}\left(x^{\prime}\right)-D_{k} u_{p}(x)\right|<\Gamma_{p}\left(r_{x x^{\prime}}\right) \text { in } E\left(\sigma_{k} \leqq \alpha_{p+1}, p=1,2, \cdots\right) \text {. }
$$

The conditions on $g(x)$ imply that for some $M_{1}^{\prime \prime},\left|D_{k} g(x)\right|<M_{1}^{\prime \prime}$ in $\bar{R}_{3}$ $\left(\sigma_{k} \leqq \alpha_{2}\right)_{\bullet} \dagger$ Say

$$
\left|D_{k} u_{p}(x)\right|<Z_{p}^{\prime} \text { in } E \quad\left(\sigma_{k} \leqq \alpha_{p+1}, p=1,2, \cdots\right) .
$$

Then as $u_{1}(x)=0$ in $R-R_{3}$, we have

$$
\left|D_{j} u_{1}\left(x^{\prime}\right) D_{l} g\left(x^{\prime}\right)-D_{j} u_{1}(x) D_{l} g(x)\right| \leqq \Gamma_{1}\left(r_{x x^{\prime}}\right) M_{1}^{\prime \prime}+Z_{1}^{\prime} \Delta_{3}\left(r_{x x^{\prime}}\right)
$$

$\dagger$ If $d_{3}$ is the diameter of $\bar{R}_{8}$, then $|g(x)|<M+\Delta_{1}\left(d_{3}\right)$ in $\bar{R}_{3}$. Now take any $k^{\prime}=\left(k_{1}, \cdots, k_{h}-1\right.$, $\left.\cdots, k_{n}\right)$ and $k=\left(k_{1}, \cdots, k_{n}\right)\left(0<\sigma_{k} \leqq \alpha_{2}\right)$. Let $x^{\prime} x^{\prime \prime}$ be a line segment parallel to the $x_{h}$-axis and lying wholly in $\bar{R}_{8}$; set $r=\left|x_{h}^{\prime \prime}-x_{h^{\prime}}\right|$. As $\left|D_{k^{\prime}} g\left(x^{\prime \prime}\right)-D_{k^{\prime}} g\left(x^{\prime}\right)\right|<\Delta_{1}(r)$, the law of the mean gives, for some point $x^{*}$ of $x^{\prime} x^{\prime \prime},\left|D_{k}\left(x^{*}\right)\right|<\Delta_{1}(r) / r$. Hence $\left|D_{k}(x)\right|<\Delta_{1}(r) / r+\Delta_{2}\left(d_{3}\right)$ in $\bar{R}_{3}\left(0<\sigma_{k} \leqq \alpha_{2}\right)$. 
for $\sigma_{j} \leqq \alpha_{2}$ and $\sigma_{l} \leqq \alpha_{2}$ and any $x$ and $x^{\prime}$ in $E$. Hence if we put

$$
\Delta_{1}^{*}(r)=\left[\left(\alpha_{2}+1\right) !\right]^{n}\left[\Gamma_{1}(r) M_{1}^{\prime \prime}+Z_{1}^{\prime} \Delta_{3}(r)\right],
$$

we shall have, on differentiating $H_{1}(x)=u_{1}(x) g(x)$,

$$
\left|D_{k} H_{1}\left(x^{\prime}\right)-D_{k} H_{1}(x)\right| \leqq \Delta_{1}^{*}\left(r_{x x^{\prime}}\right) \text { in } E \quad\left(\sigma_{k} \leqq \alpha_{2}\right) .
$$

Also $\left|D_{k} H_{1}(x)\right|<\left[\left(\alpha_{2}+1\right) !\right]^{n} Z_{1}^{\prime} M_{1}^{\prime \prime}$ in $\bar{R}_{3}$ and $=0$ in $E-R_{3}\left(\sigma_{k} \leqq \alpha_{2}\right)$; thus inequalities corresponding to (15.7) and (15.8) hold for $H_{1}(x)$. Hence if we take $\delta_{1}>0$ so that

$$
\Delta_{1}^{*}(r)<\beta_{1}^{\prime} / 2
$$

and take $\kappa_{1}$ so that

$$
1-\Phi\left(\kappa_{1} \delta_{1}\right)<\beta_{1}^{\prime} /\left\{4\left[\left(\alpha_{2}+1\right) !\right]^{n} Z_{1}^{\prime} M_{1}^{\prime \prime}\right\},
$$

then if we form $G_{1}(x)$ for any admissible $g(x)$ by means of (16.5), (16.7) will hold with $p=1$; we take $\kappa_{1}$ large enough so that the right hand side of (16.13) with $p=1$ will be $<1 / 2$.

If we differentiate (16.5) with $p=1 \sigma_{k}$ times $\left(\sigma_{k} \leqq m\right)$, we derive an equation similar to (15.5); forming this for $x=x$ and $x=x^{\prime}$ and subtracting, we find (changing $y$ to $y+x^{\prime}-x$ in one equation)

$$
D_{k} G_{1}\left(x^{\prime}\right)-D_{k} G_{1}(x)=T \kappa_{1}^{n} \int_{E}\left[D_{k} H_{1}\left(y+x^{\prime}-x\right)-D_{k} H_{1}(y)\right] e^{-\kappa_{1}^{2} r_{k y}^{2} d y}
$$

This with (17.3), (15.3), and the definition of $\Phi(\infty)$ gives

$$
\left|D_{k} G_{1}\left(x^{\prime}\right)-D_{k} G_{1}(x)\right| \leqq \Delta_{1}^{*}\left(r_{x x^{\prime}}\right) \text { in } E \quad\left(\sigma_{k} \leqq \alpha_{2}\right) .
$$

Assume now we have defined functions $\Delta_{p}{ }^{*}(r)$ and have chosen numbers $\kappa_{p}$ so that

$$
\left|D_{k} G_{p}\left(x^{\prime}\right)-D_{k} G_{p}(x)\right| \leqq \Delta_{p}^{*}\left(r_{x x^{\prime}}\right) \text { in } E \quad\left(\sigma_{k} \leqq \alpha_{p+1}\right),
$$

so that (16.7) holds, and so that the quantity in (16.13) is $<1 / 2^{p}$, for $p<q$. Then for any admissible $g(x), g(x)-\left\{G_{1}(x)+\cdots+G_{q-1}(x)\right\}$ satisfies the same kind of conditions as $g(x)$; hence, just as before, we find a function $\Delta_{q}^{*}(r)$ so that an inequality similar to (17.3) holds for $D_{k} H_{q}(x)$ in $E\left(\sigma_{k} \leqq \alpha_{q+1}\right)$. Also $H_{q}(x)$ is bounded properly; hence we can choose $\kappa_{q}$ so that (16.7) holds for any admissible $g(x)$ with $p$ replaced by $q$, and so that (16.13) with $p=q$ is $<1 / 2^{q}$. From this we show, as before, that (17.6) holds with $p$ replaced by $q$. We can thus continue finding functions $\Delta_{p}^{*}(r)$ and numbers $\kappa_{p}$ indefinitely. We put finally $G(x)=G_{1}(x)+G_{2}(x)+\cdots$, and show, just as in Lemma 6, that $G(x)$ has the required properties. This ends the proof. 


\section{Analytic extensions}

18. Proof of Theorem I. Let $g(x)$ be the extension of $f(x)$ of class $C^{m}$ given by Lemma 2. Set $R=E-A$ and define $R_{1}, R_{2}, \cdots, \alpha_{1}, \alpha_{2}, \cdots$, and numbers $\epsilon_{1}, \epsilon_{2}, \cdots$, approaching zero as in $\$ 16$. Define $G(x)$ in $E-A$ as in Lemma 6 , and set $F(x)=G(x)$ in $E-A, F(x)=f(x)$ in $A$. That $F(x)$ is of class $C^{m}$ in $E$ and property (2) holds follows from (16.1) and Lemma 1, just as in $\$ 11$; the other facts are obvious.

19. The functions $\omega_{\nu k}(x)$. In the next sections we shall discuss the analyticity of the extension of $f(x)$ at the isolated points of $A$. Let $R$ be an open set, let $a_{1}, a_{2}, \cdots$ be points of $R$ having no limit point in $R$, let $m_{1}, m_{2}, \cdots$ be corresponding integers $\geqq 0$, and let $m$ be an integer $\geqq-1$ or $\infty$. We assume that if $a_{v_{1}}, a_{v_{2}}, \ldots$ is any sequence of points $a_{\nu}$ approaching the boundary of $R$, then

$$
\lim _{s \rightarrow \infty} \inf m_{r_{0}} \geqq m \text {. }
$$

Choose about each $a_{v}$ a neighborhood $U_{v}$ lying, with its boundary, in $R$, so that no two have common points. Define the numbers $\rho(\nu ; k)$ so that when $(\nu ; k)$ runs through the values $(1 ; k), \sigma_{k} \leqq m_{1} ;(2 ; k), \sigma_{k} \leqq m_{2} ;$ etc.; then $\rho(\nu ; k)$ runs through the values $1,2,3, \ldots$. Let $\rho^{\prime}(\nu ; k)$. equal one plus the largest of the numbers $m_{1}, \cdots, m_{\nu}, \rho(\nu ; k)$.

Take any positive integer $s$, and consider all neighborhoods $U_{\nu}$ such that $\rho^{\prime}(\nu k) \geqq s$ for some $k\left(\sigma_{k} \leqq m_{\nu}\right)$; let $R_{s}$ be the set of all points of $R$ whose distances from these neighborhoods and from the boundary of $R$ are $>1 / \mathrm{s}$, and whose distances from the origin are $<s$. Then $R_{s}$ is a bounded open set, $\bar{R}_{\mathrm{s}}$ lies in $R_{s+1}(s=1,2, \cdots), R_{1}+R_{2}+\cdots=R$, and $U_{\nu}$ lies in $R-R_{p^{\prime}(p k)}$ $\left(\sigma_{k} \leqq m_{\nu}\right)$. By Lemma 2, there are functions $\omega_{\nu k}(x)$ of class $C^{\infty}$ in $E$, defined for $\sigma_{k} \leqq m_{\nu}, \nu=1,2, \cdots$, , such that

$$
D_{l} \omega_{\nu k}\left(a_{\nu}\right)=\left\{\begin{array}{l}
1, l=k \\
0, l \neq k
\end{array}\left(\sigma_{l} \leqq m_{\nu}\right) ; \omega_{\nu k}(x)=0 \text { in } E-U_{\nu}\right.
$$

Choose for each $\nu$ a positive number $\beta_{\nu}<1 / \nu$ so that $\beta_{\nu} \geqq \beta_{\nu+1}$, and

$$
\begin{aligned}
& \beta_{\nu}\left|D_{l} \omega_{\nu k}(x)\right|<1 / \nu\left[\left(m_{\nu}+1\right)^{n}\right] \text { in } E \\
&\left(\sigma_{k} \leqq m_{\nu}, \sigma_{l} \leqq m_{\nu}, \nu=1,2, \cdots\right) .
\end{aligned}
$$

Now let $f_{\nu k}$ be any set of numbers, defined for $\sigma_{k} \leqq m_{\nu}, \nu=1,2, \cdots$, satisfying the condition

$$
\left|f_{\nu k}\right| \leqq \beta_{\nu} \quad\left(\sigma_{k} \leqq m_{\nu}, \nu=1,2, \cdots\right) .
$$

Set 


$$
c_{p(\nu k)}=f_{\nu k}, \quad \omega_{p(\nu k)}^{\prime}(x)=\omega_{\nu k}(x) \quad\left(\sigma_{k} \leqq m_{\nu}, \nu=1,2, \cdots\right) .
$$

Take any $s=\rho(\nu k)$. As $\omega_{s}^{\prime}(x)=\omega_{\nu k}(x)=0$ in $R-U_{\nu}$, and $R_{\mathbf{t}}=R_{\rho(\nu k)}$ is in $R_{p^{\prime}(v k)}$ which has no points in common with $U_{v}$,

$$
\omega_{s}^{\prime}(x)=0 \text { in } R_{s} \quad(s=1,2, \cdots) .
$$

20. The transformation $L$. Define functions $u_{1}(x), u_{2}(x), \cdots$ as in Lemma 6. Consider any function

$$
g(x)=\lambda_{1} \omega_{1}^{\prime}(x)+\lambda_{2} \omega_{2}^{\prime}(x)+\cdots \quad\left(\left|\lambda_{s}\right| \leqq 1, s=1,2, \cdots\right) ;
$$

such functions and $\lambda$ 's we shall call admissible. Set

$$
\epsilon_{s}=\beta_{s} / 2^{s+1} \quad(s=1,2, \cdots) .
$$

There are, obviously, functions $\Delta_{1}(r), \Delta_{2}(r), \cdots$, so that (17.1) holds for any such $g(x)$; hence, by Lemma 7 , we can define numbers $\kappa_{1}, \kappa_{2}, \cdots$, so that if $G(x)$ is defined in terms of $g(x)$ as in Lemma 6, then $G(x)$ is analytic in $R$ and (16.1) holds. In using Lemma 6, we replace $\alpha_{p}$ by $p$.

We note here a certain property of $G(x):$ If $g(x)$ is admissible and

$$
\text { if } g(x)=0 \text { in } R_{s} \text {, then }\left|D_{k} G(x)\right|<\epsilon_{s-1} / 2^{s-2} \text { in } \bar{R}_{\mathrm{s}-1} \quad\left(\sigma_{k} \leqq s-1\right) \text {. }
$$

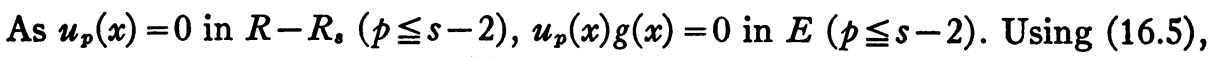
we see in succession that $G_{1}(x) \equiv 0, \cdots, G_{s-2}(x) \equiv 0$. This with (16.9) and (16.11) gives

$$
\left|D_{k} G(x)\right| \leqq \sum_{p=s-1}^{\infty}\left|D_{k} G_{p}(x)\right|<\sum_{p=s-1}^{\infty} \epsilon_{p} / 2^{p} \leqq \epsilon_{s-1} / 2^{s-2}
$$

in $\bar{R}_{t-1}\left(\sigma_{k} \leqq s-1\right)$, as required.

Given any admissible $g(x)$, let $L g(x)$ be the corresponding function $G(x)$. It follows easily from the definition of $G(x)$ that $L$ is linear:

$$
L\left[\lambda_{1} g_{1}(x)+\lambda_{2} g_{2}(x)\right]=\lambda_{1} L g_{1}(x)+\lambda_{2} L g_{2}(x) .
$$

We show now that for admissible numbers $\lambda$,

$$
L \sum_{s=1}^{\infty} \lambda_{s} \omega_{s}^{\prime}(x)=\sum_{s=1}^{\infty} \lambda_{s} L \omega_{s}^{\prime}(x) .
$$

To prove this, take any point $x^{0}$ of $R$, in the set $R_{q}$, and any $\epsilon>0$. Take $q^{\prime} \geqq q$ so that $1 / 2^{q^{\prime}-8}<\epsilon$. (19.6) and (20.3), for $s=q^{\prime}+1, q^{\prime}+2, \cdots$, give, as $\lambda_{0} \omega_{\bullet}^{\prime}(x)$ is admissible,

$$
\left|\sum_{s=q^{\prime}+1}^{\infty} \lambda_{s} L \omega_{s}^{\prime}(x)\right|=\left|\sum_{s=q^{\prime}+1}^{\infty} L \lambda_{s} \omega_{s}^{\prime}(x)\right|<\sum_{s=q^{\prime}+1}^{\infty} 1 / 2^{s-2}=1 / 2^{q^{\prime}-2}<\epsilon / 2
$$


in $R_{q^{\prime}}$, and in particular, at $x^{0}$. As

$$
\sum_{s=q^{\prime}+1}^{\infty} \lambda_{s} \omega_{\ell}^{\prime}(x)=0 \text { in } R_{q^{\prime}+1}
$$

and is admissible,

$$
\left|L \sum_{:=q^{\prime}+1}^{\infty} \lambda_{\imath} \omega_{\varepsilon}^{\prime}\left(x^{0}\right)\right|<1 / 2^{q^{\prime}-1}<\epsilon / 2 .
$$

Moreover

$$
L \sum_{\rho=1}^{\infty} \lambda_{s} \omega_{\rho}^{\prime}(x)=\sum_{s=1}^{q^{\prime}} \lambda_{s} L \omega_{s}^{\prime}(x)+L \sum_{s=q^{\prime}+1}^{\infty} \lambda_{s} \omega_{s}^{\prime}(x)
$$

hence

$$
\begin{aligned}
& \left|L \sum_{s=1}^{\infty} \lambda_{s} \omega_{s}^{\prime}\left(x^{0}\right)-\sum_{s=1}^{\infty} \lambda_{s} L \omega_{s}^{\prime}\left(x^{0}\right)\right| \\
& \quad \leqq\left|L \sum_{s=q^{\prime}+1}^{\infty} \lambda_{s} \omega_{s}^{\prime}\left(x^{0}\right)\right|+\left|\sum_{s=q^{\prime}+1}^{\infty} \lambda_{s} L \omega_{s}^{\prime}\left(x^{0}\right)\right|<\epsilon
\end{aligned}
$$

which proves (20.5).

We prove two inequalities. Take any $(\nu ; k),(\mu ; l)\left(\sigma_{k} \leqq m_{\nu}, \sigma_{l} \leqq m_{\mu}\right)$; then

$$
\begin{aligned}
& \left|D_{k} L \omega_{\mu l}\left(a_{v}\right)-D_{k} \omega_{\mu l}\left(a_{v}\right)\right|<\epsilon_{\rho(v k)}, \\
& \left|D_{k} L \omega_{\mu l}\left(a_{v}\right)-D_{k} \omega_{\mu l}\left(a_{v}\right)\right|<\epsilon_{\rho(\mu l)} .
\end{aligned}
$$

The first follows from (16.1) when we note that $a_{\nu}$ is in $R-R_{\rho^{\prime}(v k) \text {, and }}$ $\epsilon_{\rho^{\prime}(\nu k)}<\epsilon_{\rho(\nu k)}$, and $\sigma_{k} \leqq m_{\nu}<\rho^{\prime}(\nu k)$ (recall that $\alpha_{p}$ was replaced by $p$ in using Lemma 6). We now prove the second. As $\omega_{\mu l}(x)=0$ in $R_{\rho^{\prime}(\mu l)}$ and $\rho^{\prime}(\mu l)$ $\geqq \rho(\mu l)+1,(20.3)$ gives

(a) $\left|D_{k^{\prime}} L \omega_{\mu l}(x)-D_{k^{\prime}} \omega_{\mu l}(x)\right|<\epsilon_{\rho(\mu l)}$ in $\bar{R}_{\rho^{\prime}(\mu l)-1} \quad\left(\sigma_{k^{\prime}} \leqq \rho^{\prime}(\mu l)-1\right)$.

Also (16.1) gives

(b) $\left|D_{k^{\prime}} L \omega_{\mu l}(x)-D_{k^{\prime}} \omega_{\mu l}(x)\right|<\epsilon_{\rho(\mu l)}$ in $R-R_{p} \quad\left(\sigma_{k^{\prime}} \leqq p, p \geqq \rho^{\prime}(\mu l)-1\right)$.

Say $a_{\nu}$ is in $R_{p+1}-R_{p}$. As $a_{\nu}$ is not in $R_{\rho^{\prime}(\nu k)}, \rho^{\prime}(\nu k) \leqq p$, and $\sigma_{k} \leqq m_{\nu} \leqq \rho^{\prime}(\nu k)$ $-1 \leqq p-1$. If $p \geqq \rho^{\prime}(\mu l)-1$, (20.7) follows directly from (b). If $p<\rho^{\prime}(\mu l)-1$, then $a_{v}$ is in $R_{\rho^{\prime}(\mu)-1}$, and $\sigma_{k} \leqq p-1<\rho^{\prime}(\mu l)-1$, and (a) applies.

21. An infinite system of linear equations. We prove here

Lemma 8. Suppose $\eta_{s}$ and $c_{s}(s=1,2, \cdots)$, and $\gamma_{s t}(s, t=1,2, \cdots)$, are given, so that $1 \geqq \eta_{s} \geqq \eta_{s+1}>0(s=1,2, \cdots),\left|c_{s}\right| \leqq 1$, and

$$
\left|\gamma_{s t}\right|<\eta_{s} / 2^{t+1} \quad(s, t=1,2, \cdots) .
$$


Then there are numbers $\lambda_{s}(s=1,2, \cdots)$ such that

$$
\sum_{t=1}^{\infty}\left(\gamma_{s t}+\delta_{s t}\right) \lambda_{t}=\sum_{t=1}^{\infty} \gamma_{s t} \lambda_{t}+\lambda_{s}=c_{s} \quad(s=1,2, \cdots),
$$

and

$$
\left|\lambda_{\mathbf{s}}-c_{\mathbf{\imath}}\right| \leqq \eta_{\bullet}
$$$$
(s=1,2, \cdots)
$$

Using the method of successive approximations, put

$$
\lambda_{1 s}=c_{s}, \quad \lambda_{p s}=-\sum_{t=1}^{\infty} \gamma_{s t} \lambda_{p-1, t} \quad(p=2,3, \cdots) .
$$

It is readily proved by induction that

$$
\left|\lambda_{p s}\right|<\eta_{s} / 2^{p-1} \quad(p=2,3, \cdots) .
$$

Hence the series $\lambda_{1 s}+\lambda_{2 s}+\cdots$ converges to a limit $\lambda_{s}(s=1,2, \cdots)$, and

$$
\begin{gathered}
\sum_{t=1}^{\infty}\left(\gamma_{s t}+\delta_{s t}\right) \lambda_{t}=\sum_{p=1}^{\infty} \sum_{t=1}^{\infty}\left(\gamma_{s t}+\delta_{s t}\right) \lambda_{p t}=\sum_{p=1}^{\infty}\left(\lambda_{p s}-\lambda_{p+1, s}\right)=c_{s}, \\
\left|\lambda_{s}-c_{s}\right|=\left|\sum_{p=2}^{\infty} \lambda_{p s}\right| \leqq \sum_{p=2}^{\infty} \eta_{s} / 2^{p-1}=\eta_{s} .
\end{gathered}
$$

22. We are now ready to prove

LEMMA 9. Let $R, m, a_{v}, m_{\nu}(\nu=1,2, \ldots)$ be defined as in $\$ 19$. Then there are numbers $\beta_{v}>0(\nu=1,2, \cdots)$ with the following property. Given any set of numbers $f_{v k}$ defined for $\sigma_{k} \leqq m_{\nu}, \nu=1,2, \cdots$, such that (19.4) holds, there exists a function $G(x)$ analytic in $R$, such that

$$
D_{k} G\left(a_{\nu}\right)=f_{\nu k} \quad\left(\sigma_{k} \leqq m_{\nu}, \quad \nu=1,2, \cdots\right),
$$

and such that if we set $G(x) \equiv 0$ in $E-R$, then $G(x)$ is of class $C^{m}$ in $E$, and

$$
D_{k} G(x)=0 \text { in } E-R \quad\left(\sigma_{k} \leqq m\right) .
$$

We define the $\omega_{\nu k}(x)$ and the $\beta_{\nu}$ as in $\$ 19$. Now take any $f_{\nu k}$ satisfying (19.4), and define the $c_{\rho(v k)}$ by (19.5). Define the $\epsilon_{s}$ and the transformation $L$ as in $\$ 20$. Set

$$
\eta_{p(\nu k)}=\beta_{\nu} \quad\left(\sigma_{k} \leqq m_{\nu}, \nu=1,2, \cdots\right),
$$

and

(22.4) $\gamma_{s t}=\gamma_{\rho(\nu k) \rho(\mu l)}=D_{k} L \omega_{\mu l}\left(a_{\nu}\right)-\delta_{s t}=D_{k} L \omega_{\mu l}\left(a_{v}\right)-D_{k} \omega_{\mu l}\left(a_{v}\right)$.

Let $u=\rho(\theta j)$ be the larger of the two numbers $s=\rho(\nu k), t=\rho(\mu l)$. Then using (20.6) or (20.7) according as $u=s$ or $u=t$, we find (as $\left.\beta_{\rho(\theta)} \leqq \beta_{\rho(\nu k)} \leqq \beta_{v}\right)$ 


$$
\left|\gamma_{s t}\right|<\epsilon_{\rho(\theta j)} \leqq \beta_{v} / 2^{u+1} \leqq \eta_{\theta} / 2^{t+1} .
$$

Also $\left|c_{s}\right|=\left|f_{\nu k}\right| \leqq \beta_{\nu}<1$. Therefore the equations (21.2) have a solution $\lambda_{1}, \lambda_{2}, \cdots$, and

$$
\sum_{t=1}^{\infty}\left(\gamma_{s t}+\delta_{s t}\right) \lambda_{t}=\sum_{\mu, l} \lambda_{\rho(\mu l)} D_{k} L \omega_{\mu l}\left(a_{\nu}\right)=c_{\theta}=c_{\rho(\nu k)}=f_{\nu k} .
$$

By (22.8) below, the $\lambda$ 's are admissible ( $\$ 20)$, and we can define the analytic function $G(x)$ in $R$ by the equation

$$
G(x)=L \sum_{t=1}^{\infty} \lambda_{t} \omega_{t}^{\prime}(x)
$$

(20.5) and (22.5) give

$$
D_{k} G\left(a_{\nu}\right)=D_{k} \sum_{t=1}^{\infty} \lambda_{t} L \omega_{t}^{\prime}\left(a_{\nu}\right)=\sum_{\mu, l} \lambda_{\rho(\mu l)} D_{k} L \omega_{\mu l}\left(a_{\nu}\right)=f_{\nu k} .
$$

(19.6) and (20.3) show that the last sum above is uniformly convergent in any $\bar{R}_{p}$; hence the termwise differentiation is permissible.

Set $G(x) \equiv 0$ in $E-R$; we must show that $G(x)$ is of class $C^{m}$ in $E$. (This is trivial if $m=-1$.) First note that, by (19.4) and (21.3),

$$
\left|\lambda_{\rho(\nu k)}\right| \leqq\left|c_{\rho(\nu k)}\right|+\eta_{\rho(\nu k)}=\left|f_{\nu k}\right|+\beta_{\nu} \leqq 2 \beta_{p} ;
$$

this with (19.3) gives (replacing $\nu, k$ and $l$ by $\mu, l$ and $k$ )

$$
\begin{array}{r}
\left|\lambda_{\rho(\mu l)} D_{k} \omega_{\mu l}(x)\right|<2 /\left[\mu\left(m_{\mu}+1\right)^{n}\right] \text { in } E \\
\left(\sigma_{k}, \sigma_{l} \leqq m_{\mu}, \mu=1,2, \cdots\right) .
\end{array}
$$

Now take any boundary point $x^{0}$ of $R$, any integer $m^{\prime} \leqq m$, and any $\epsilon>0$. Take $q \geqq m^{\prime}$ so that $\epsilon_{q}<\epsilon / 2$. Take $\delta>0$ so that $R_{q}$ has no points within $\delta$ of $x^{0}$, and so that if $\nu$ is any number such that $U_{\nu}$ has points within $\delta$ of $x^{0}$, then $m_{\nu} \geqq m^{\prime}$ and $2 / \nu<\epsilon / 2$ (see (19.1)). Consider any point $y$ of $R$ within $\delta$ of $x^{0}$; and take any $k, \sigma_{k} \leqq m^{\prime}$. Either $D_{k} \omega_{\mu l}(y)=0$ for all $\mu, l$, or else for some $\mu, y$ lies in $U_{\mu}$, in which case there are at $\cdot \operatorname{most}\left(m_{\mu}+1\right)^{n}$ such numbers $\neq 0$, and $2 / \mu<\epsilon / 2$, and $m_{\mu} \geqq m^{\prime}$. Thus if we replace $x$ by $y$ in (22.9) and sum over $\mu$ and $l$, we find

$$
\left|D_{k} \sum_{t=1}^{\infty} \lambda_{t} \omega_{t}^{\prime}(y)\right|=\left|\sum_{\mu, l} \lambda_{\rho(\mu l)} D_{k} \omega_{\mu l}(y)\right|<\epsilon / 2 \quad\left(\sigma_{k} \leqq m^{\prime}\right) .
$$

Ás $y$ is in $R-R_{q}$, replacing $\alpha_{q}$ by $q \geqq m^{\prime}$ in (16.1) gives

$$
\left|D_{k} L \sum_{t=1}^{\infty} \lambda_{t} \omega_{t}^{\prime}(y)-D_{k} \sum_{t=1}^{\infty} \lambda_{t} \omega_{t}^{\prime}(y)\right|<\epsilon_{q}<\epsilon / 2 \quad\left(\sigma_{k} \leqq m^{\prime}\right) .
$$


This with the last inequality gives

$$
\left|D_{k} G(y)\right|<\epsilon \text { in } R \text { if } r_{x^{\circ} y}<\delta \quad\left(\sigma_{k} \leqq m^{\prime}\right) ;
$$

the proof is completed with the aid of Lemma 1.

23. Functions analytic at the isolated points of $A$. Lemmas 4, 6 and 9 lead directly to the following theorem.

Theorem II. Let $A$ be a closed set in $E$, and let $a_{1}, a_{2}, \cdots$ be isolated points of $A$. Set $A^{\prime}=A-\left(a_{1}+a_{2}+\cdots\right)$. Let $m$ be an integer $\geqq-1$ or $\infty$, and let the integers $m_{\nu} \geqq 0, \nu=1,2, \cdots$, satisfy (19.1). Let $f_{k}(x)$ be defined for $x$ in $A^{\prime}\left(\sigma_{k} \leqq m\right)$, and for $x=a_{v}\left(\sigma_{k} \leqq m_{v}\right)$, so that $f(x)$ is of class $C^{m}$ in $A$. Then there is a function $F(x)$ of class $C^{m}$ in $E$ such that

(1) $F(x)=f(x)$ in $A$,

(2) $D_{k} F(x)=f_{k}(x)$ in $A^{\prime}$ for $\sigma_{k} \leqq m$ and at each $a_{\nu}$ for $\sigma_{k} \leqq m_{\nu}$,

(3) $F(x)$ is analytic in $E-A^{\prime}$.

We asked that $f(x)$ be of class $C_{*}^{m}$ in $A$, while $f_{k}\left(a_{v}\right)$ may not be defined for certain values of $\nu$ and $k\left(\sigma_{k} \leqq m\right)$. We require merely that after setting $f_{k}\left(a_{v}\right)=0\left(\sigma_{k}>m_{v}\right), f(x)$ shall be of class $C^{m}$ in $A$.

A special case of interest is $m=-1$. The $m_{\nu}$ and the $f_{k}\left(a_{v}\right)$ are then unrestricted. $A^{\prime}$ may be void, in which case $f(x)$ is analytic throughout $E$. $A^{\prime}$ may of course contain isolated points.

To prove the theorem, set $R=E-A^{\prime}$ and determine the open sets $R_{\nu}$ and the numbers $\beta_{\nu}(\nu=1,2, \cdots)$, as in $\$ 19$. Let $g^{\prime}(x)$ be the extension of $f(x)$ of class $C^{m}$ in $E$ and of class $C^{\infty}$ in $E-A^{\prime}$ given by Lemma 4 (setting $f_{k}\left(a_{v}\right)=0$ for $\sigma_{k}>m_{v}$ ). Let $G^{\prime}(x)$ be the analytic function in $R$ given by Lemma 6 (with $\alpha_{p}$ replaced by $p$ ) such that

$$
\left|D_{k} G^{\prime}(x)-D_{k} g^{\prime}(x)\right|<\beta_{p} \text { in } R-R_{p} \quad\left(\sigma_{k} \leqq p\right),
$$

and set $G^{\prime}(x)=f(x)$ in $A^{\prime} . G^{\prime}(x)$ is of class $C^{m}$ in $E$, and

$$
D_{k} G^{\prime}(x)=f_{k}(x) \text { in } A^{\prime}
$$$$
\left(\sigma_{k} \leqq m\right)
$$

(see §18). Set

$$
f_{\nu k}=D_{k} g^{\prime}\left(a_{\nu}\right)-D_{k} G^{\prime}\left(a_{\nu}\right) \quad\left(\sigma_{k} \leqq m_{\nu}, \nu=1,2, \cdots\right) .
$$

As $a_{\nu}$ lies in $R-R_{\rho^{\prime}(\nu k)}$ and $\rho^{\prime}(\nu k)>m_{\nu},(23.1)$ gives $\left|f_{\nu k}\right|<\beta_{\rho^{\prime}(\nu k)}<\beta_{\nu}\left(\sigma_{k} \leqq m_{\nu}\right)$. Thus the conditions of Lemma 9 are satisfied, and there is a function $G(x)$ analytic in $R, \equiv 0$ in $A^{\prime}$, of class $C^{m}$ in $E$, and such that (22.1) and (22.2) hold. Set

$$
F(x)=G^{\prime}(x)+G(x)
$$


then $F(x)$ is our required function. It is of class $C^{m}$ in $E$ as the same is true of $G^{\prime}(x)$ and $G(x)$; it is analytic in $R=E-A^{\prime}$ as the same is true of $G^{\prime}(x)$ and $G(x)$; it equals $f(x)$ in $A^{\prime}$ as $G^{\prime}(x)=f(x)$ and $G(x)=0$ there. (22.1), (23.3) and (14.2) show that $D_{k} F\left(a_{v}\right)=D_{k} g^{\prime}\left(a_{v}\right)=f_{k}\left(a_{v}\right)\left(\sigma_{k} \leqq m_{v}\right) ;(22.2)$ and (23.2) show that $D_{k} F(x)=f_{k}(x)$ in $A^{\prime}$, completing the proof.

24. An extension-approximation theorem. We prove here

ThEOREM III. $\dagger$ Let $A$ be closed, and let $A_{-1}, A_{0}, A_{1}, \cdots$ be closed subsets of $A$ such that each $A_{s}$ lies in $A_{s+1}$. Let $a_{s 1}, a_{s 2}, \cdots$ be points of $A_{s}-A_{s-1}$ which are isolated points of $A$, and set $A^{\prime}=A-\sum a_{s t}$. Let $B_{-1}$ be void, and let $B_{0}$, $B_{1}, \cdots$ be sets whose sum $B$ lies in $E-A$, such that each $B_{s}$ lies in $B_{s+1}$, such that each set $B_{s}-B_{s-1}$ has limit points in $B-B_{s-1}+A_{s}$ only, and such that each set $A+B-B_{s}$ is closed. Let $f_{k}(x)$ be defined in each set $\Gamma_{s}=A+B-\left(A_{s-1}+B_{s-1}\right)$ for $\sigma_{k} \leqq s(s=0,1, \cdots)$ so that $f(x)=f_{0}(x)$ is of class $C^{*}$ in $\Gamma_{s}$ in terms of the $f_{k}(x)$ for each s. Let $\epsilon(x)$ be a continuous function, positive in $E-A^{\prime}$ and zero in $A^{\prime}$. Then there is a function $F(x)$ defined in $E-A_{-1}$ such that

(1) $F(x)$ is of class $C^{s}$ in $E-A_{s-1}(s=0,1, \ldots)$,

(2) $D_{k} F(x)=f_{k}(x)$ in $A-A_{s-1}\left(\sigma_{k} \leqq s, s=0,1, \cdots\right)$,

(3) $\left|D_{k} F(x)-f_{k}(x)\right|<\epsilon(x)$ in $B-B_{s-1}\left(\sigma_{k} \leqq s, s=0,1, \cdots\right)$,

(4) $F(x)$ is analytic in $E-A^{\prime}$.

Any number of sets $A_{s}, B_{s}$ may be void; any of the points $a_{s t}$ may not exist. Note that if $A_{\infty}=A-\left(A_{-1}+A_{0}+\cdots\right)$, then $F(x)$ is of class $C^{\infty}$ at all points of $A_{\infty}$. Theorem I for $m$ finite is obtained by letting $B$ and $A_{-1}, \cdots$, $A_{m-1}$ be void, and setting $A=A_{m}$; and for $m$ infinite, by letting $B$ and every $A_{s}$ be void. Theorem II is obtained similarly; we arrange the $a_{s t}$ in a sequence $a_{1}, a_{2}, \cdots$, and set $m_{\nu}=s$ if $a_{\nu}$ is in $A_{s}-A_{s-1}$. Lemma 6 is obtained by setting $A=A_{-1}=E-R, B_{s}=R_{s+1}(s=0,1, \ldots)$, and taking $\epsilon(x)$ so that $\epsilon(x) \leqq \epsilon_{s}$ in $R-R_{8}$.

We turn now to the proof. Take a subdivision of the open set $E-(A+B)$ as in $\S 8$, let $y^{0 v}(\nu=1,2, \ldots)$ be the vertices of the cubes, and let $x^{0 \nu}$ be a point of $\Gamma_{0}$ whose distance from $y^{0 \nu}$ is not more than twice the distance from $y^{0 v}$ to $\Gamma_{0}$. Define the functions $\phi_{0 v}(x)$ in $E-(A+B)$ as in $\$ 9$, and define $g_{0}^{\prime}(x)$ by (11.1), using the functions $\phi_{0 \nu}(x)$ and $\psi_{0 ; 0}\left(x ; x^{0 \nu}\right)=f\left(x^{0 \nu}\right)$, and replacing $E-A$ and $A$ by $E-(A+B)$ and $\Gamma_{0}$ respectively. Then $g_{0}^{\prime}(x)$ is defined throughout $E-A_{-1}$, and is easily seen to be a continuous extension of $f(x)$. Let $g_{0}(x)$ be a function $=g_{0}^{\prime}(x)$ in $A-A_{-1}+\Gamma_{1}$ and analytic in the open set $E-\left(A+\Gamma_{1}\right)$ so that

$\dagger$ A special case of this theorem has been proved by A. Besikowitsch, Über analytische Funktionen mit vorgeschriebenen Werten ihrer Ableitungen, Mathematische Zeitschrift, vol. 21 (1924), pp. 111-118. 
(24.1)

$$
\left|g_{0}(x)-g_{0}^{\prime}(x)\right|<\theta_{1}(x) / 4 \text { in } E-\left(A+\Gamma_{1}\right),
$$

where $\theta_{p}(x)=\min \left[\epsilon(x)\right.$, distance from $x$ to $\left.A+\Gamma_{p}\right](p=1,2, \cdots)$. Then $g_{0}(x)$ is continuous in $E-A_{-1}$.

We shall now define in succession functions $g_{1}(x), g_{2}(x), \cdots$, with the following properties:

(a) $g_{p}(x)$ is defined in $E-A_{-1}$, is of class $C^{s}$ in $E-A_{s-1}(s=0, \cdots, p)$, and is analytic in $E-\left(A+\Gamma_{p+1}\right)$.

(b) $D_{k} g_{p}(x)=f_{k}(x)$ in $A-A_{s-1}+\Gamma_{p+1}\left(\sigma_{k} \leqq s, s=0, \cdots, p\right)$. $p-1)$.

(c) $\left|D_{k} g_{p}(x)-D_{k} g_{p-1}(x)\right|<\epsilon(x) / 2^{p+2}$ in $B_{p-1}-B_{s-1}\left(\sigma_{k} \leqq s, s=0, \cdots\right.$,

(d) $\left|D_{k} g_{p}(x)-f_{k}(x)\right|<\epsilon(x) / 2^{p+2}$ in $B_{p}-B_{p-1}\left(\sigma_{k} \leqq p\right)$.

Assuming $g_{0}(x), \cdots, g_{p-1}(x)$ are defined, we shall define $g_{p}(x)$. Consider any point of $\Gamma_{p}$; it is at a positive distance from the closed set $A_{p-1}$, and hence we can enclose it in an open set lying at a positive distance from $A_{p-1}$. We thus enclose $\Gamma_{p}$ in an open set $\Gamma_{p}^{\prime}$ containing no points of $A_{p-1}$, and having no limit points in $A_{p-1}$ other than limit points of $\Gamma_{p}$. Take a subdivision of the open set $E-\left(A+\Gamma_{p}\right)$, let $y^{p \nu}(\nu=1,2, \ldots)$ be the vertices of the cubes, and let $x^{p v}$ be a point of $\Gamma_{p}$ whose distance from $y^{p v}$ is not more than twice the distance from $y^{p \nu}$ to $\Gamma_{p}(\nu=1,2, \cdots)$. Define the functions $\phi_{p v}(x)$ in $E-\left(A+\Gamma_{p}\right)$ as in $\S 9$ and define $\psi_{p ; k}\left(x^{\prime} ; x\right)$ by $(6.1)\left(\sigma_{k} \leqq p\right)$, replacing $m$ by $p$. Remembering that $f(x)$ is of class $C^{p}$ in $\Gamma_{p}$, set

$$
g_{p}^{\prime}(x)=\sum_{\nu} \phi_{p \nu}(x) \psi_{p ; 0}\left(x ; x^{p \nu}\right) \text { in } \Gamma_{p}^{\prime}-\Gamma_{p},
$$

and set $g_{p}^{\prime}(x)=g_{p-1}(x)$ in $\Gamma_{p}$. From the proof in $\$ 11$ it is seen that $g_{p}^{\prime}(x)$ is an extension of class $C^{p}$ of the values of $f(x)$ in $\Gamma_{p}$.

Set $\zeta_{p}(x)=g_{p}^{\prime}(x)-g_{p-1}(x)$ in $\Gamma_{p}^{\prime}$; then $\zeta_{p}(x)$ is of class $C^{p-1}$ in $\Gamma_{p}^{\prime}$, and

$$
D_{k} \zeta_{p}(x)=0 \text { in } \Gamma_{p} \quad\left(\sigma_{k} \leqq p-1\right) .
$$

Set $\eta_{p}=1 /\left\{2^{p+4} c[(p+1) !]^{n}\left(36 n^{1 / 2}\right)^{p} N^{(p)}\right\} \quad(p=0,1, \cdots)$, where $N^{(p)}=$ $\max N_{k}$ for $\sigma_{k} \leqq p$. Let $K_{p-1}$ be the set of points of $B_{p-1}$ for which

$$
\left|D_{k} \zeta_{p}(x)\right| \geqq \eta_{p-1} \epsilon(x) \delta_{x}^{p-1-\sigma_{k}} \text { for some } k \quad\left(\sigma_{k} \leqq p-1\right),
$$

where $\delta_{x}$ is the distance from $x$ to $\Gamma_{p}$, or 1 if that is smaller. Each point of $A-A_{p-1}$ is at a positive distance from $K_{p-1}$, as $B_{p-1}$ has no limit points in $A-A_{p-1}$, and each point of $B-B_{p-1}$ is at a positive distance from $K_{p-1}$ on account of (24.3), as $\epsilon(x)>0$ in $B$; hence each point of $\Gamma_{p}$ is at a positive distance from $K_{p-1}$, and we can enclose $\Gamma_{p}$ in an open set $\Gamma_{p}^{\prime \prime}$ which lies in $\Gamma_{p}^{\prime}$ and contains no points of $K_{p-1}$. Now 


$$
\left|D_{k} \zeta_{p}(x)\right|<\eta_{p-1} \epsilon(x) \delta_{x^{p-1-\sigma_{k}}} \text { in } \Gamma_{p}^{\prime \prime} \cdot B_{p-1}
$$$$
\left(\sigma_{k} \leqq p-1\right) \text {. }
$$

We can also take $\Gamma_{p}^{\prime \prime}$ so that if $\rho_{p}(x)$ is the distance from $x$ to $A_{p-1}$, then

$$
\left|D_{k} \zeta_{p}(x)\right|<\eta_{p-1} \rho_{p}(x) \delta_{x}^{p-1-\sigma_{k}} \text { in } \Gamma_{p}^{\prime \prime} \quad\left(\sigma_{k} \leqq p-1\right) .
$$

Let $I_{\nu_{t}}$ be those sets $I_{\nu}$ of the subdivision of $E-\left(A+\Gamma_{p}\right)$ lying wholly in $\Gamma_{p}^{\prime \prime}(t=1,2, \cdots)$, and set

$$
g_{p}^{\prime \prime}(x)=g_{p-1}(x)+\zeta_{p}(x) \sum_{t=1}^{\infty} \phi_{p_{t}}(x) \text { in } E-\left(A+\Gamma_{p}\right),
$$

and $g_{p}^{\prime \prime}(x)=g_{p-1}(x)=f(x)$ in $A-A_{-1}+\Gamma_{p}$. Then $g_{p}^{\prime \prime}(x)$ is of class $C^{p}$ in $E-A_{p-1}$, and with the help of (24.4) we find

$$
\left|D_{k} g_{p}^{\prime \prime}(x)-D_{k} g_{p-1}(x)\right|<\epsilon(x) / 2^{p+3} \text { in } B_{p-1} \quad\left(\sigma_{k} \leqq p-1\right)
$$

(see \$13). Also (24.5) gives

$$
\left|D_{k} g_{p}^{\prime \prime}(x)-D_{k} g_{p-1}(x)\right|<\rho_{p}(x) / 2^{p+3} \text { in } E-A \quad\left(\sigma_{k} \leqq p-1\right),
$$

and hence $g_{p}^{\prime \prime}(x)$ is of class $C^{i}$ in $E-A_{a_{-1}}(s=0, \cdots, p)$, as the same is true of $g_{p-1}(x)(s=0, \cdots, p-1)$.

Finally let $g_{p}(x)$ be an analytic function such that

$$
\left|D_{k} g_{p}(x)-D_{k} g_{p}^{\prime \prime}(x)\right|<\theta_{p+1}(x) / 2^{p+3} \text { in } E-\left(A+\Gamma_{p+1}\right) \quad\left(\sigma_{k} \leqq p\right) ;
$$

set $g_{p}(x)=g_{p-1}(x)=f(x)$ in $A-A_{-1}+\Gamma_{p+1}$. Then $g_{p}(x)$ has all the required properties. (c) is a direct consequence of the above inequality and (24.7); (d) follows from (24.9) and the fact that $D_{k} g_{p}^{\prime \prime}(x)=f_{k}(x)$ in $\Gamma_{p}\left(\sigma_{k} \leqq p\right)$; (a) and (b) follow with the aid of Lemma 1.

Set

$$
g(x)=\lim _{p \rightarrow \infty} g_{p}(x) \text { in } E-A_{-1} .
$$

By (24.8) and (24.9), $g(x)$ exists and is of class $C^{\infty}$ in $E-A$. Let $x$ be any point of any $A_{0}-A_{\theta_{-1}}$; by (a), $g_{p}(x)$ is of class $C^{*}$ in a neighborhood of $x$ for $p \geqq s$, and by (24.8) and (24.9), the same is true of $g(x)$. The same argument, using (b), shows that

$$
D_{k} g(x)=f_{k}(x) \text { in } A-A_{s-1}\left(\sigma_{k} \leqq s, s=0,1, \cdots\right) .
$$

Finally (c), (d), (24.1) and the definition of $g_{0}^{\prime}(x)$ show that

$$
\left|D_{k} g(x)-f_{k}(x)\right|<\epsilon(x) / 2 \text { in } B-B_{s-1} \quad\left(\sigma_{k} \leqq s, s=0,1, \cdots\right) .
$$

We have now found an extension $g(x)$ with all the properties but (4). It is replaced by an analytic extension $F(x)$ just as in $\$ 23$; we must be careful merely to make 


$$
\left|D_{k} F(x)-D_{k} g(x)\right|<\epsilon(x) / 2 \text { in } B-B_{s-1}\left(\sigma_{k} \leqq s, s=0,1, \cdots\right) .
$$

Let $a_{1}, a_{2}, \cdots$ be the $a_{s t}$ arranged in a sequence, and set $m_{\nu}=s$ if $a_{\nu}$ is in $A_{\varepsilon}-A_{\mathrm{s}-1}$. Set $R=E-A^{\prime}$. Let $R_{p}$ consist of those points of the $R_{p}$ of $\$ 19$ whose distances from the closed set $A+B-B_{p-1}$ are $>1 / p(p=1,2, \cdots)$. Every point of $E-A^{\prime}$ lies in some $R_{p}$, as $B-\left(B_{0}+B_{1}+\cdots\right)$ is void. Take the $\beta_{\nu}(\$ 19)$ small enough so that if $\left|\lambda_{\rho(v k)}\right| \leqq 2 \beta_{\nu}$ (see (22.8)) and $g^{*}(x)$ $=\sum \lambda_{t} \omega_{t}^{\prime}(x)$, then

$$
\left|D_{k} g^{*}(x)\right|<\epsilon(x) / 8 \text { in } R_{p+1}-R_{p} \quad\left(\sigma_{k} \leqq p\right) .
$$

Let $\epsilon_{s}^{\prime}$ be one eighth the lower bound of $\epsilon(x)$ for $x$ in $R_{s+1}(s=1,2, \cdots)$, or 1 if that is smaller. Replace the $\epsilon_{s}$ of $(20.2)$ by $\min \left(\epsilon_{s}, \epsilon_{s}^{\prime}\right)$. Then for any such $g^{*}(x),(16.1)$ gives

$$
\left|D_{k} L g^{*}(x)\right|<\epsilon_{p}+\left|D_{k} g^{*}(x)\right|<\epsilon(x) / 4 \text { in } R_{p+1}-R_{p} \quad\left(\sigma_{k} \leqq p\right) .
$$

Replace the $g^{\prime}(x)$ of $\$ 23$ by the present $g(x)$, and determine $G^{\prime}(x)$ so that

$$
\left|D_{k} G^{\prime}(x)-D_{k} g(x)\right|<\min \left[\beta_{p}, \epsilon(x) / 4\right] \text { in } R-R_{p}
$$$$
\left(\sigma_{k} \leqq p\right) \text {, }
$$

and, in particular, in $B-B_{p-1}$. Now if $G(x)$ and $F(x)$ are determined as in $\$ 23$, then (24.13) and hence property (3) hold; the other properties are easily verified, and the proof of the theorem is complete.

HaRVARD UNIVERSity,

Cambridge, Mass. 